\title{
1 Asian wild apples threatened by gene flow from domesticated apples and by
}

\section{2 their pestified pathogen}

3 Alice Feurtey ${ }^{1,2, a}$, Ellen Guitton ${ }^{3, a}$, Marie De Gracia Coquerel $^{3}$, Ludovic Duvaux ${ }^{3,4}$, Jason

4 Shiller $^{3,5}$, Marie-Noëlle Bellanger ${ }^{3}$, Pascale Expert ${ }^{3}$, Mélanie Sannier ${ }^{3}$, Valérie Caffier ${ }^{3}$,

5 Tatiana Giraud ${ }^{1}$, Bruno Le $\mathrm{Cam}^{3}$ and Christophe Lemaire ${ }^{3^{*}}$

$6 \quad{ }^{1}$ Ecologie Systématique Evolution, CNRS, AgroParisTech, Université Paris-Saclay, 91400

7 Orsay, France

$8 \quad{ }^{2}$ Max Planck Institute for Evolutionary Biology, 24306 Plön, Germany

$9 \quad{ }^{3}$ IRHS-UMR1345, Université d'Angers, INRAE, Institut Agro, SFR 4207 QuaSaV, 49071,

10 Beaucouzé, France

$11 \quad{ }^{4}$ BIOGECO, INRAE, Université de Bordeaux, 33610 Cestas, France

$12 \quad{ }^{5}$ Noble Research Institute, Ardmore, OK 73401, USA

13 a Alice Feurtey and Ellen Guitton should be considered joint first author

$14 *$ Correspondence : Dr. Christophe Lemaire. IRHS, 42 rue G. Morel. 49071 Beaucouzé

15 Cedex, France.

17 E-mail: christophe.lemaire@ univ-angers.fr 


\section{Abstract}

21 Massive gene flow between crops and their wild relatives may threaten the genetic integrity of wild species. Such threats are now well documented, but little is known about indirect consequences involving the spillover of crop pathogens to wild plants or introgression between crop and wild pathogens. To address these questions, we used population genetics approaches, demographic inference and pathogenicity tests on host-pathogen pairs composed of wild or domesticated apple trees of Central Asia and their fungal pathogen, Venturia inaequalis, itself showing differentiated agricultural-type and wild-type populations. We confirmed the occurrence of gene flow from cultivated to wild apple trees in Asian forests, threatening the Asian wild apple genetic integrity. SNP markers and demographic modeling revealed the occurrence of a secondary contact followed by hybridization between agricultural-type and wild-type fungal pathogen populations, and the dispersal of the agricultural-type pathogen in wild forests. We detected a SNP predicting the ability of the fungus to parasitize the different host populations, which induced an early stop codon in a gene coding for a small secreted protein in the agricultural-type fungal population, thus representing a putative avirulence gene which function loss would enable to parasitize cultivated apples. Pathogenicity tests in fact revealed the pestification of $V$. inaequalis, with higher virulence of the agricultural-type population on both wild and domesticated trees. Our findings highlight the threat posed by cultivating a crop near its center of origin, with the invasion of a pestified pathogen on wild plants and introgression in the wild-type pathogen.

40 Keywords: apple, crop-to-wild gene flow, hybridization, Malus, secondary contact, 41 avirulence gene, Venturia 
bioRxiv preprint doi: https://doi.org/10.1101/2020.04.15.042242; this version posted April 16, 2020. The copyright holder for this preprint (which was not certified by peer review) is the author/funder. All rights reserved. No reuse allowed without permission.

43 


\section{$44 \quad$ Introduction}

45 The domestication of plants corresponds to genetic and phenotypic differentiation between 46 crops and their wild relatives under human selection (Zeder, Emshwiller, Smith \& Bradley,

47 2006). However, crops and their wild relatives often remain interfertile, which can lead to 48 introgression when the two taxa remain geographically close or come into secondary contact 49 (Ellstrand et al., 2013). The hybrids resulting from crop-to-wild pollination events may have a 50 low fitness in natural environments (Wang, Viera, Crawford, Chu \& Nielsen., 2017), 51 potentially driving to extinction the wild lineages receiving massive gene flow from its 52 relative crop (Todesco et al., 2016; Wolf, Takebayashi \& Rieseberg, 2001). In contrast, hybrids can outcompete pure wild individuals, thus threatening the genetic integrity of wild lineages (Feurtey, Cornille, Shykoff, Snirc \& Giraud, 2017; Hooftman, Jong, Oostermeijer \& Den Nijs, 2007; Hovick, Campbell, Snow \& Whitney, 2012), thereby jeopardizing future adaptation to global changes and leading to a loss of valuable genetic resources for breeding 57 programs.

A much less widely studied, but just as alarming, consequence of secondary contact between crops and their wild relatives, is the possible gene flow between their respective pathogens, which are themselves often closely related. Such gene flow between differentiated pathogen populations or species parasitizing wild and domesticated hosts can promote the emergence of new diseases or the breakdown of resistance, through the generation of pathogens with an expanded host range (Depotter, Seidl, Wood \& Thomma, 2016), higher resistance to 64 antibiotics (Hanage, Fraser, Tang, Connor \& Corander, 2009) or enhanced virulence (i.e. degree of damage caused by the pathogen to its host) (Stukenbrock, Christiansen, Hansen, Dutheil \& Schierup, 2012), thus potentially representing a major threat to both crop health 
and wild host persistence (Lemaire et al., 2016; Leroy, Lemaire, Dunemann \& Le Cam, 2013; Leroy et al., 2016). Pathogen spillover (i.e., infection by a pathogen on another host than its endemic host) and hybridization between closely related phytopathogenic fungal species following secondary contact have been reported in several systems (Feurtey \& Stukenbrock, 2018; Gladieux et al., 2011; Stukenbrock, 2016 a, b). Indeed, when fungal populations have diverged in allopatry and have specialized on new hosts and later met in sympatry (i.e., secondary contact), pre-mating barriers are often weak (Giraud, Refrégier, Le Gac, de Vienne \& Hood, 2008; Le Gac \& Giraud, 2008) and they can hybridize and/or still infect their ancestral host. The resulting spillover and hybridization events can lead to disease emergence and/or increased disease severity (Ioos, Andrieux, Marçais \& Frey, 2006; Lin et al., 2007; Newcombe, Stirling, McDonald \& Bradshaw, 2000; Stukenbrock et al., 2012). In anther-smut Microbotryum fungi for example, two sister castrating pathogens parasitize two sister plant species and co-occur in sympatry as a consequence of a secondary contact following allopatric divergence (Gladieux et al., 2011). One of the two species, Microbotryum lychnidisdioicae (DC. ex Liro, Deml \& Oberw.), is however better at infecting both hosts, and spillovers occur in nature (de Vienne, Hood \& Giraud, 2009; Gladieux et al., 2011). In poplars, hybrid trees were generated and planted as they were resistant to the two rust species parasitizing the two parental tree species. However, a fungal hybrid between the two rust species rapidly emerged that was able to cause disease on the hybrid poplars (Newcombe et al., 2000). Yet, the consequences of crop-to-wild gene flow on the evolution of wild pathogen populations and on the disease of their wild host has been little investigated. Joint analyses of both wild and agricultural hosts and pathogens are required for a comprehensive understanding of the consequences of secondary contacts of crops and their wild relatives, and of their pathogens, as well as for elucidating coevolutionary histories and local adaptation. 
91 Yet, such studies combining both host and pathogen population genetic analyses remain 92 scarce (Croll and Laine 2016).

93 Here, we addressed these questions on apple trees and their apple scab fungal pathogen, 94 Venturia inaequalis (Cooke) G. Winter, which represent good models for investigating the consequences of secondary contact between crops and their wild relatives. They indeed have both wild and agricultural differentiated species/populations that have recently been reunified in their center of origin and may thus possibly hybridize (Figure 1). The cultivated apple (Malus domestica Borkh.) is the most cultivated fruit tree of temperate areas worldwide (FAO STATS from 2008-2018, as accessed on 04-07-2020). Analysis of phenotypic diversity, genetic markers, archeological data and demographic inference showed that the cultivated apple was initially domesticated from $M$. sieversii Ledeb. M. Roem (Figure 1A), forming quasi-monospecific natural forests of wild apple in the Tian Shan Mountains in Central Asia (Cornille et al., 2012; Harris, Robinson \& Juniper, 2002; Vavilov, 1931; Velasco et al., 2010 ), which have been declared a World Heritage Center by UNESCO. The cultivated apple was imported into Europe by the Romans via the Caucasian and Mediterranean coasts, crossing the distribution ranges of various wild apple tree species on the way, leading to major secondary contributions to the cultivated apple tree genepool from several crabapples (Cornille et al., 2012; Cornille, Gladieux \& Giraud 2013; Nikiforova, Cavalieri, Velasco \& Goremykin, 2013). There has been in particular, in Western Europe, an important contribution from the European crabapple, M. sylvestris (L.) Mill, to the M. domestica genome (Figure 1A). Domestication and breeding has led to high differentiation between $M$. domestica and its

112 various wild progenitors (Cornille et al., 2012). However, because apple species generally 113 have weak interspecific barriers, introgression can still occur between wild and cultivated 
114 forms (Cornille et al., 2012; Nikiforova et al., 2013). This situation raises serious concerns

115 regarding possible gene flow and its deleterious consequences for the conservation of 116 important wild genetic resources. Yet, most studies on gene flow between domesticated and 117 wild apples have focused on the European wild apple tree. These studies have shown that 118 extensive crop-to-wild gene flow is threatening the genetic integrity of the European 119 crabapple M. sylvestris (Cornille et al., 2015; Feurtey et al. 2017; Figure 1A). In Central Asia, 120 at the end of the 19th century, western European $M$. domestica apple varieties have been planted in orchards close to M. sieversii forests, leading to recent secondary contact between M. domestica and M. sieversii. A couple of studies have suggested the occurrence of gene flow from $M$. domestica to the Asian crabapple $M$. sieversii, although without investigating the contact sites that orchards represent (Cornille et al. 2012; Omasheva et al., 2017). Such gene flow may affect the genetic integrity of $M$. sieversii, which would amplify the current threat on Asian wild-apple forest ecosystems, which are already endangered by forest destruction, $M$. sieversii being included in the International Union for Conservation of Nature's Red List of Threatened Species (IUCN Red List) as a vulnerable organism.

Apple domestication has also fostered divergence in the ascomycete fungus $V$. inaequalis, a haploid fungus with an obligatory sexual reproduction event each year. This fungus is responsible for the apple scab disease, producing gray-brown lesions on leaves and fruits, and leading to major economic losses on cultivated apples. Venturia inaequalis parasitizes $M$. domestica and several wild apple tree species, such as $M$. sieversii, M. sylvestris and $M$. 134 floribunda (Siebold ex Van Houtte), and differentiated populations occur on these different 135 Malus species (Figure 1B; Gladieux et al., 2010b). Apple trees and V. inaequalis share a 136 common geographical origin in Central Asia (Cornille et al., 2012; Gladieux et al., 2008), M. 
137 sieversii being the wild host of origin of the fungus (Gladieux et al., 2010b). In the Central

138 Asian Mountains, in forests where M. domestica is absent, a wild-type $V$. inaequalis 139 population that represents a relic of the ancestral population has been found on M. sieversii 140 trees (Gladieux et al., 2010b). An agricultural-type $V$. inaequalis population has been found in 141 Central Asia (Gladieux et al., 2010b) in the peri-urban or agricultural environments on $M$. 142 domestica and on M. sieversii (Figure 1B). These wild-type and agricultural-type populations 143 of $V$. inaequalis began diverging in Central Asia between 2,000 and 4,000 years ago 144 (Gladieux et al., 2010b), the agricultural-type population having then spread into Europe together with the domesticated apple (Figure 1B; Gladieux et al., 2010b). It has not been studied yet whether the current co-occurrence of the agricultural-type and wild-type $V$. inaequalis populations represents a secondary contact between the two fungal populations, and what are its epidemiological consequences through potential spillover and introgressions (Wang et al., 2017). Indeed, gene flow between wild-type and agricultural-type $V$. inaequalis populations may lead to the emergence of hybrids harboring new epidemiological traits, potentially harmful to both wild and cultivated apple trees, and/or to the dispersal of the agricultural-type population into wild forests. As a matter of fact, previous studies have shown that the $V$. inaequalis population parasitizing $M$. floribunda, and highly differentiated from all the populations on $M$. sieversii and M. sylvestris, migrated to resistant varieties of $M$. domestica, which promoted a resistance breakdown and gene flow between pathogen populations (Figure 1B; Lemaire et al. 2016; Leroy et al. 2016).

157 Despite the importance of wild Asian apple trees as an endangered wild species and as a 158 valuable genetic resource for future breeding programs, the potential impacts of the secondary 159 contact between domesticated and wild apple trees in Central Asia on the fitness of both the 
160 plant and its associated pathogen have not been investigated yet. The lack of diagnostic 161 morphological features for apple species makes the use of genetic markers necessary for 162 species and hybrid identification (Cornille, Giraud, Smulders, Roldán-Ruiz \& Gladieux, 163 2014). We therefore used microsatellite markers in apple trees and genome-wide single164 nucleotide polymorphisms (SNPs) in $V$. inaequalis, together with pathogenicity assays, to 165 assess the occurrence and impact of secondary contact on both fruit trees and their fungal 166 pathogens in their center of diversity. First, we investigated whether the introduction of 167 domesticated apple trees in Central Asia threatened the genetic integrity of $M$. sieversii wild apple trees via introgressions, and whether orchards planted in forests represent particular threats. We then assessed whether the agricultural-type fungal population occurred on wild apple trees and hybridized with the wild-type population. We tested, using demographic modeling and scenarios comparison, the hypothesis that the co-occurrence and hybridization 172 of agricultural-type and wild-type $V$. inaequalis populations resulted from a secondary contact at the time when domesticated trees were introduced in orchards near wild apple forests, after a period of allopatry. We also tested whether some genomic regions in $V$. inaequalis were good predictors of its ability to parasitize the different host populations. Finally, we tested, using experimental inoculations, whether such gene flow increased the threats to the wild apple tree through increase in virulence of the pathogen populations.

179 Material and Methods

\section{Sampling}

181 Plant and fungal materials were sampled in 2012 at nine sites in Kazakhstan in the Tian Shan 
183 dominated by wild apple trees (Cornille et al., 2014). Seven of the nine sampling sites were 184 located in natural apple forests, farther than dozens of kilometers from any orchard. The two 185 remaining sampling sites corresponded to a 20-30 year-old orchard and the wild natural forest 186 immediately surrounding the orchard, respectively. We sampled apple trees in the orchard site 187 and wild apple trees in the surrounding forest. This orchard was situated directly in the middle 188 of the natural forest and constituted a rare situation allowing to investigate the consequences 189 of the secondary contact for both the host and the pathogen. We obtained plant material from 245 apple trees, originating from the nine sites. The 245 plant samples corresponded to 185 leaves and 60 fruit skin pieces, all harbouring visible scab lesions (Table S1). No morphological differences between $M$. domestica and $M$. sieversii allow to distinguish them reliably in the field. Therefore, all sampled trees were genotyped with 33 microsatellite markers and were assigned to $M$. domestica or $M$. sieversii species or to a hybrid class using as references 50 apple genotypes previously characterized as being non-hybrid $M$. domestica genotypes and 25 apple genotypes previously characterized as being non-hybrid $M$. sieversii genotypes (Cornille et al., 2012).

Monospore isolation of the fungus was performed from disease lesions on 205 of these 245 trees: 24 to $48 \mathrm{~h}$ after spreading a spore suspension obtained from the diseased material on a Petri dish with malt-agar medium, we took a single germinated spore using a needle under a stereomicroscope for culture on a new Petri dish. We performed one monospore isolation per tree for 148 trees and two to four monospore isolations per tree for 57 trees. In total, we obtained 269 strains (Table S1). In addition to the fungal collection sampled for this study, we

204 included in our final dataset $15 \mathrm{~V}$. inaequalis strains previously assigned to the pure wild type 205 population and $18 \mathrm{~V}$. inaequalis strains previously assigned to the agricultural-type population 206 (Gladieux et al., 2010b). These 33 V. inaequalis reference strains whose genomes have been 
207 previously sequenced (Le Cam et al., 2019) had been sampled in Kazakhstan on M. sieversii, 208 either from a peri-urban environment near Almaty $\left(43^{\circ} 15000 \mathrm{~N}-76^{\circ} 54000 \mathrm{E}\right)$ or from a natural 209 forest site in the Tian Shan Mountains $\left(43^{\circ} 13807\right.$ N-7 $\left.7^{\circ} 16783 \mathrm{E}\right)$. Despite being sampled on

$210 M$. sieversii, the 18 strains from the peri-urban environment clustered with strains sampled on 211 domesticated apples in Kazakh orchards, thus belonging to the agricultural type (Gladieux et 212 al., 2010b) (Figure 1B; Table S2).

SNP design and genotyping for the apple scab fungus Venturia inaequalis

We used the set of single nucleotide polymorphisms (SNPs) previously detected in the genomes of the 15 wild-type and 18 agricultural-type reference strains (Gladieux et al., 2010b; Le Cam et al., 2019). SNPs were filtered in order to avoid transposable elements and AT-rich regions, as well as more than $20 \%$ of missing data (Le Cam et al., 2019). After filtering, the dataset contained 1,210,121 SNPs, with no bias of missing data frequencies between wild and agricultural populations. In order to identify a set of SNPs distinguishing agricultural and wild-type $V$. inaequalis strains, we selected 192 SNPs among the 1,210,121 available SNPs as those meeting three requirements: $i)$ with moderate $\left(F_{S T}=0.21\right)$ to high $\left(F_{S T}=1\right)$ differentiation between wild-type and agricultural-type reference populations (Figure S1, Table S3), to obtain reasonable statistical power to assign genotypes to wild, agricultural and hybrid types, ii) physically distant enough to ensure lack of linkage disequilibrium between them (correlation coefficient between markers within population $\mathrm{r}^{2}<$

227 0.1), and iii) found in predicted genes (Le Cam et al., 2019). 
230 S3) using the KASPAR method (KBioscience, competitive allele-specific polymerase chain

231 reaction assay). KASPAR genotyping was performed at the Gentyane platform (INRA,

232 Clermont-Ferrand, France) using $10 \mathrm{ng}$ of haploid DNA mixed with the KASP genotyping 233 master mix (Catalogue number: KBS-1016-017, LGC Genomics, Hoddesdon, UK) and 234 custom KASP SNP assays (LGC Genomics, Hoddesdon, UK). Only SNPs and individuals 235 with less than $20 \%$ missing data were kept for analyses. After filtering for missing data, the 236 dataset was composed of 255 fungal strains genotyped for 181 SNPs. Details about SNP 237 location on the $V$. inaequalis reference genome are given in Table S3.

\section{Genotyping using microsatellite markers for the host apple tree}

240 In order to assign trees to wild, domesticated and hybrid types, all apple trees were genotyped using microsatellite markers. Apple tree DNA was extracted from leaves with the NucleoSpin plant DNA extraction kit II® (Macherey \& Nagel). Microsatellite PCR amplifications were performed with a Multiplex PCR Kit® (QIAGEN, Inc.). To genotype the 245 trees, we used 33 microsatellite markers spread across the 17 chromosomes using 10 different multiplex reactions, as previously described (Cornille et al., 2012). The genotyping was done at the Gentyane platform (INRA, Clermont-Ferrand, France). Only the microsatellite markers with less than $30 \%$ of missing data were used and only individuals with less than $50 \%$ of missing data were kept for the analyses. The final dataset was thus composed of 240 apple tree samples genotyped at 28 microsatellite markers.

Since fungi were sampled on the genotyped host trees, we had genotypes for host and pathogen pairs. We obtained genotypes for 249 host-pathogen pairs, several fungal samples originating from the same trees (132 trees with one fungal strain, 49 trees with two strains,

253 five trees with three strains and one tree with four strains). 
255 Analyses of diversity, differentiation and hybridization

256 Reference genotypes of $M$. domestica $(\mathrm{N}=50)$ and $M$. sieversii $(\mathrm{N}=25)$ (Cornille et al., 2012)

257 were used to estimate the proportion of ancestry from these two species in the 240 genotyped

258 apple trees. We computed a hybrid index $(H I)$ implemented in the add-on R-package

259 Introgress (Gompert \& Buerkle, 2010), an estimate of the proportion of alleles that were

260 inherited from one of the two parental populations, i.e. the level of ancestry in one of two

261 populations. In our case, we set pure $M$. domestica trees to $H I=1$ while $M$. sieversii had $H I=0$.

262 This index therefore represents the proportion of ancestry in the M. domestica genepool (thus

263 called here $\left.P_{d o m}\right)$. For the pathogen, we used the same package to compute a hybrid index

264 based on the reference genotypes of strains previously identified as belonging to the

265 agricultural-type population $(\mathrm{N}=18)$ or to the wild-type $(\mathrm{N}=15)$ population (Gladieux et al.,

$2662010 b$ ). Here, we set $H I=1$ for the agricultural type, so that the hybrid index represents the

267 proportion of ancestry in the agricultural-type population (here called $P_{\text {agr }}$ ) genepools.

In most of our analyses, we used the hybrid indices as continuous values, and not discrete classes, to avoid potential biases generated by arbitrary thresholds. Only for the analyses of apple and fungal population or species spatial distribution, of the genetic determinants of adaptation and of pathogenicity tests, we assigned apple and fungal genotypes to discrete classes. Apple tree genotypes with a $P_{d o m}$ index below 0.2 and those with a $P_{d o m}$ index greater than 0.8 were considered to belong to $M$. sieversii and $M$. domestica, respectively, based on the distribution of the hybrid index (Figure 2A). A fungal strain with a $P_{a g r}$ below 0.1 was considered to be a wild-type strain, whereas a strain with a $P_{a g r}$ greater than 0.9 was

276 considered to be an agricultural-type strain, based on the distribution of the hybrid index 277 (Figure 2B). The threshold was more stringent for fungi than for trees, as more markers were 
278 available and the power was, therefore, higher. For both pathogens and hosts, genotypes with 279 intermediate hybrid index values were considered to be hybrids. Assigning hybrids to more 280 precise hybrid classes (ie F1, F2 or backcrosses) is not possible with the current dataset for 281 haploid organisms such as $V$. inaequalis. When we used discrete classes for analyses, we 282 checked that the choice of the thresholds did not affect the inference.

283 For both apple and $V$. inaequalis samples, we also investigated genetic differentiation and 284 species or genetic lineage assignment through principal component analyses (PCAs) using the 285 ade4 R package (Dray \& Dufour, 2007) based on the multilocus genotypes (microsatellite markers for trees and SNPs for fungi). We further investigated the existence of population 287 structure within apple tree species and within the previously delimited $V$. inaequalis populations using the software STRUCTURE v.4.3 (Falush, Stephens \& Pritchard, 2003) based on 10 runs of 250,000 Monte Carlo Markov Chain (MCMC) iterations after a burn-in of 25,000 iterations using the admixture model and assuming a number of clusters ranging from 1 to 6. The output of STRUCTURE was processed with Clumpp (Jakobsson \& Rosenberg, 2007) and we used the method described in Evanno et al. (2005) and implemented in STRUCTURE HARVESTER (Earl \& vonHoldt, 2012) to detect the strongest population subdivision level. We tested the significance of correlations between the hybrid degrees inferred from coordinate values of the first PCA axis, the hybrid index and the ancestry probability given by STRUCTURE using a Pearson's product-moment correlation coefficient in $\mathrm{R}$.

For both apple and $V$. inaequalis populations, we estimated classical population genetics statistics, i.e., expected heterozygosity $\left(H_{e}\right.$, Weir \& Cockerham, 1984), as well as global and pairwise differentiation $F_{S T}$ (Weir \& Cockerham, 1984) between the nine sampling sites. All 
302

303

304

305

306

307

308

309

310

311

312

313

314

315

316

317

318

319

320

321

322

323

Significativity of global and pairwise $F_{S T}$ was assessed using Fisher's exact tests of allele frequency differences between sampling sites with 10,000 dememorization and batches and 10,000 iterations per batch. We also performed Mantel tests of correlation (Mantel, 1967) between pairwise $F_{S T}$ matrices computed for apple and $V$. inaequalis populations on the one hand, and between each pairwise $F_{S T}$ matrix and a matrix of geographical distance between sampling sites on other hand, using the mantel.test function of the ape v5.3 $\mathrm{R}$ package (Paradis \& Schliep, 2019).

\section{Genetic footprints of host adaptation in Venturia inaequalis}

In order to detect genetic signatures of adaptation to host species in $V$. inaequalis populations, we performed a discriminant analysis on principal components (DAPC; Jombart, Devillard \& Balloux, 2010) implemented in the R package adegenet v.2.12 (Jombart, 2008). Unlike PCA which focuses on overall genetic variance, this procedure seeks variables, the discriminant functions, that maximize differences between a priori delimited groups, while minimizing the variance within these groups. We applied this analysis by defining groups in $V$. inaequalis based on the assignment of their apple tree groups of collection (M. domestica, M. sieversii and hybrids). This approach also aims at detecting the genetic factors (here SNPs) that contributed the most to this ecologically-based clustering. If one of the 181 SNPs in $V$. inaequalis was closely linked to a genetic variant involved in adaptation to host species, its loading on the first discriminant functions would indeed be greater than that of other SNPs. In addition, we estimated monolocus pairwise $F_{S T}$ between the three host-based fungal clusters, using Genepop v4.7.5 (Rousset, 2008). 
324 To assess what allele was ancestral or derived at the SNP found to predict the host species of

325 collection, we used five available genomes (Le Cam et al., 2019) belonging to the outgroup $V$.

326 inaequalis lineage parasitising Pyracantha (Gladieux, Caffier, Devaux \& Le Cam, 2010a; Le

327 Cam, Parisi \& Arene, 2002), with the following NCBI accession numbers SAMN07816619,

328 SAMN07816620, SAMN07816621, SAMN07816617, SAMN07816618, corresponding to the 329 strains 186, 1669, 2266, 2269 and 2507 respectively (Le Cam et al., 2019).

\section{Pathogenicity tests of $V$. inaequalis on $M$. domestica and $M$. sieversii}

In order to test for an effect of the agricultural, hybrid or wild-type status of $V$. inaequalis strains on their pathogenicity on $M$. domestica and $M$. sieversii, we performed artificial inoculations using 57 strains from our sampling, corresponding to 20 strains identified as agricultural-type, 17 strains identified as wild-type and 20 hybrid strains (Table S1). We used one variety of $M$. domestica $\left(\mathrm{GALA}^{\circledR}, \mathrm{X} 4712\right)$ and one accession of $M$. sieversii (GMAL 3619.b) for the pathogenicity tests. Gala is a variety planted worldwide despite being highly susceptible to apple scab. GMAL 3619.b has been collected from the Tarbagatai mountain range by American apple breeders (Forsline, Aldwinckle, Dickson, Luby \& Hokanson, 2003) and then introduced in France. We grafted the plants on the rootstock MM106 to obtain a vigorous growth favorable to disease development and we inoculated $V$. inaequalis strains when the plants were actively growing. Prior to inoculation, grafted plants were transferred to a quarantine-controlled climatic chamber. Because of the Asian origin of the strains, we used a quarantine chamber to prevent the risk of pathogen escape. Due to the lack of space in climatic chambers, we had to perform experiments at two different dates. In the first experiment, we used 10 agricultural-type strains, 10 hybrids and 8 wild-type strains. In the 
347 second experiment, we used 10 agricultural-type strains, 10 hybrids and 9 wild-type strains. In

348 addition, for normalizing the two experiments, six strains (two strains of each type) that were

349 used in the first experiment were also included in the second experiment and used as 350 calibration strains in the analysis.

351 Each strain of $V$. inaequalis was grown on a cellophane sheet placed on malt-agar medium at $35217^{\circ} \mathrm{C}$ to obtain spores (Caffier et al., 2014). The cellophane sheets were dried and stored at $35320^{\circ} \mathrm{C}$. A spore suspension was made by collecting spores from these cellophane sheets and 354 diluting them in water to a final concentration of $1.5 \times 10^{5}$ spores $\mathrm{mL}^{-1}$ (Lê Van et al., 2012). 355 For each strain, the spore suspension was inoculated on three Gala trees and three GMAL 356 3619.b trees using a mechanical air pressure sprayer. As trees actively grew during the 357 experiment, we labelled the youngest fully deployed leaf of each tree as the F0 leaf, one or 358 two days prior to inoculation to facilitate later disease scoring. To favor spore germination 359 and fungal infection, the plants were kept in darkness with moisture maintained at $100 \%$ and 360 temperature at $17^{\circ} \mathrm{C}$ for 48 hours after inoculation (Lê Van et al., 2012). Afterward, moisture 361 was reduced to $80 \%$ during the day and $90 \%$ at night, with 12 hours of light per day. These 362 climatic conditions are highly favourable to scab infection and thus provide a good indication 363 of the strain ability to infect tree genotypes (MacHardy, 1996). For each host genotype, plants 364 were randomized within three blocks to have in each block one host replicate for each strain. 365 Disease severity, i.e., virulence of the fungal strain, was measured when there was no more 366 increase of the disease symptoms: at 19 days post inoculation (dpi) for $M$. sieversii and at 21 367 dpi for $M$. domestica. Disease severity was measured as the percentage of a leaf displaying 368 sporulation, from $0 \%$ when there was no disease symptom to $100 \%$ when the whole leaf was 369 covered with spores. The F0 leaf being the last leaf deployed one or two days prior to 370 inoculation, the assessment was done on the F0 leaf and on the first leaf under F0, named F1. 


\section{Statistical analysis of phenotypic data}

373 The data of the two distinct inoculation experiments were standardized based on the data from

374 the six calibration strains: the disease severity values were corrected to obtain the same mean

375 between the two distinct experiments for the six calibration strains. The virulence of each

376 strain was estimated as the mean percentage of scabbed leaf area across the two leaves F0 and

377 F1 and across the three inoculated plants of a given genotype (either M. domestica or $M$.

378 sieversii), so that there was one value per fungal strain and per apple tree genotype in the

379 analysis. As the assumptions of normality were not met, we compared the medians of

380 virulence between the three different populations (agricultural-type, wild-type and hybrids)

381 using a Kruskal-Wallis and a post-hoc Wilcoxon test at $21 \mathrm{dpi}$ on M. domestica and 19 dpi on

382 M. sieversii in R (version 3.4.4; R Core Team, 2018), considering the different strains in each

383 population as replicates for the population effect. In addition, a Kendall test was performed to

384 analyse the correlation between virulence and hybrid index $P_{a g r}$ for the 20 hybrid strains.

Demographic inference for wild and agricultural types of the apple scab fungus Venturia

inaequalis

In order to test demographic scenarios, and in particular the likelihood of divergence history without gene flow followed by a secondary contact in the fungal pathogen, we used the composite-likelihood, diffusion approximation-based approach for demographic inference implemented in $\partial \mathrm{a} \partial \mathrm{i}$ (Gutenkunst, Hernandez, Williamson \& Bustamante, 2009). We used the 
thinned the 1,210,121 available SNPs for these genomes to keep only one SNP every $5 \mathrm{~kb}$ (a threshold set based on the LD decay curve, Figure S2) using vcftools (Danecek et al., 2011), which left 6,187 SNPs. Using $\partial a \partial \partial$ (Gutenkunst et al., 2009), we tested a set of four basic models (Tine et al., 2014): strict isolation (SI) in which the two lineages diverge without gene flow, isolation with migration (IM) in which the two lineages diverge with constant gene flow, ancient migration (AM) in which the two lineages diverge with gene flow and stop exchanging genes at a time noted $\mathrm{T}_{\mathrm{AM}}$, and secondary contact $(\mathrm{SC})$ in which the two lineages diverge without gene flow and then exchange gene since a secondary contact occurring at $\mathrm{T}_{\mathrm{SC}}$. Each of these four models was evaluated and fitted with the observed joint allele frequency spectrum (jAFS) using 20 independent runs. Each run started with perturbed starting parameters, from which a global optimisation was done with a simulated annealing optimisation procedure and was followed by an optimisation phase with a maximum number of 100 iterations (https://popgensealab.wordpress.com/dadi-inference/; Christe et al., 2017; Tine et al., 2014). For nested models, likelihood ratio tests (LRTs) were used to identify the best model. For non-nested models, the relative likelihood of Akaike criterion (AIC) was used instead (Christe et al., 2017).

Demographic parameters were estimated for the four models. These parameters included migration rates in the two directions, effective sizes in the two lineages, divergence time and time elapsed since other demographic events ( $\mathrm{T}_{\mathrm{AM}}$ in $\mathrm{AM}$ model and $\mathrm{T}_{\mathrm{SC}}$ in $\mathrm{SC}$ model). The ancestral effective size before the split was calculated as $N_{r e f}=\theta /(2 \times \mu \times L)$ (Gutenkunst et al., 2009), where $\theta$ was estimated by $\partial \mathrm{a} \partial \mathrm{i} ; \mu$ is the mutation rate per nucleotide per generation, estimated to be $2 \times 10^{-8}$ from an analysis of divergence with the closely related species Venturia pirina parasitizing pear trees (Le Cam et al., 2019; data not shown). $L$ is the effective genome length analyzed, being computed as G x s/S (Gutenkunst et al., 2009), where $G$ is the 
418 size of the genome used, $S$ is the total number of SNPs called in $G$, and $s$ the actual number of 419 SNPs used for inference. In our analysis, 1,210,121 SNPs were called on a portion of 34,942 $420 \mathrm{Mb}$ of the genome, 6,187 SNPs were used, so that $L$ was computed as $34,942,000 \times(6,187 /$ $4211,210,121)=178,648$ bp. All other parameters were scaled by $N_{\text {ref }}$. Times in years were 422 computed using a generation time of one year, as $V$. inaequalis undergoes an obligate sexual 423 reproduction once a year. The model parameters estimated and their $95 \%$ confidence intervals 424 were obtained with Godambe methods (Coffman, Hsieh, Gravel \& Gutenkunst, 2015) from 4251000 bootstraps across SNPs.

\section{$427 \quad$ Results}

428 Contrasting distributions of the wild and agricultural host and pathogen populations in the Tian Shan Mountains

430 We explored the genetic makeup of apple trees and their scab pathogens in a wild 431 environment, by sampling apple trees and fungal strains from the same trees, at nine sites in 432 the forests of the Tian Shan Mountains in Kazakhstan. For apple trees, the PCA (Figure 2A) 433 and STRUCTURE analysis (Figures S3A and B) confirmed the existence of two genetically 434 distinct clusters corresponding to the orchard trees on the one hand, assigned to M. domestica, 435 and most of the wild forest trees on the other hand, assigned to $M$. sieversii. We did not detect 436 further population subdivision within the wild species (Figures 2A and S3). Both the first axis 437 of the PCA and STRUCTURE analyses revealed footprints of admixture between the two tree 438 species. The $P_{d o m}$ hybrid index values were also estimated for the 240 successfully genotyped 439 trees. All three methods gave congruent results (Figs. 2A and S3C). The correlation 440 coefficient between $P_{d o m}$ and the coordinates of the first PCA axis was high and significant 
$441 \quad(\mathrm{r}=-0.97 ; \mathrm{p}$-value $<0.001 ;$ Figure $\mathrm{S} 4 \mathrm{~A})$. The correlations between the ancestry proportion 442 inferred by STRUCTURE and $P_{d o m}$ on the one hand, and between the ancestry proportion 443 inferred by STRUCTURE and the coordinates of the first axis of the PCA on the other hand, 444 were also high and significant $(\mathrm{r}=0.96$, p-value $<0.001$, and $\mathrm{r}=-0.97$, $\mathrm{p}$-value $<0.001$, 445 respectively). Based on the $\mathrm{P}_{\text {dom }}$ hybrid index, we identified $78 \%$ pure wild $M$. sieversii trees, 446 ca. $12 \%$ M. domestica trees and near $10 \%$ hybrid trees (Figure 2A; Table S1). The species 447 identified genetically were consistent with our expectations during sampling: only one $M$. 448 domestica was identified in the natural forest sites (Figure 3), whereas most of the trees 449 sampled in the orchard belonged to M. domestica (28 out of 30 trees sampled in the orchard, 450 with 14 different genotypes). Three of the forest sites contained only $M$. sieversii trees, but the 451 four other forest sites contained both M. sieversii and hybrid trees (with hybrid trees 452 representing 11.1 to $27.6 \%$, Figures 3 ), as did the forest site directly surrounding the orchard 453 (with hybrid trees reaching there 27.3\%).

454 For V. inaequalis, the PCA (Figure 2B) and STRUCTURE analysis (Figures S5A and B) 455 confirmed the existence of two differentiated populations and the lack of further subdivision, 456 and revealed admixture between the wild and agricultural populations (Figures 2B and S5B).

457 The hybrid index $P_{a g r}$, the ancestry coefficient given by STRUCTURE and the first axis of the 458 PCA, separating individuals according to their degree of ancestry into the two divergent 459 populations (Figure 2B), gave congruent results (Figure S5C). The correlation coefficient 460 between $\mathrm{P}_{\mathrm{agr}}$ and the first axis of the PCA was high and significant $(\mathrm{r}=0.99 ; \mathrm{p}$-value=0.001) 461 (Figure S4B). The correlations between the ancestry proportion inferred by STRUCTURE 462 and $P_{a g r}$ on the one hand, and between the ancestry proportion inferred by STRUCTURE and 463 the coordinates of the first axis of the PCA on the other hand, were also high and significant 464 (r=0.99, p-value $<0.001$ and $r=0.98, \mathrm{p}$-value $<0.001$, respectively). 
465 The $P_{a g r}$ hybrid index estimated for the $255 \mathrm{~V}$. inaequalis strains displayed a U-shaped 466 distribution, with 33 fungal strains (12.9\%) having $P_{a g r}$ values between 0.1 and 0.9 , thus being 467 considered to be hybrids, and with high frequencies of pure agricultural-type (38.5\%) and 468 pure wild-type (48.6\%) fungal genotypes (Figure 2B; Table S1). All the fungal strains 469 sampled in the orchard carried alleles typical of the agricultural-type (Figure 3), and the 470 fungal strains sampled on the surrounding forest were mostly either agricultural-type or wild471 type genotypes. A single hybrid strain was detected in the forest surrounding the orchard, 472 whereas $3.4 \%$ to $37 \%$ of the fungal strains were hybrids in the other forest sites (Figure 3 ).

473 In order to further explore the relationship between host and pathogen genotypes, we 474 compared their genetic diversity and differentiation across the sampling sites. Genetic 475 diversities per sampling site ranged from 0.703 (tuk) to 0.802 (kot) for apple trees and from 4760.244 (tuk) to 0.487 (kot) for $V$. inaequalis (Table S4). Genetic diversities were minimal and 477 maximal in the same sampling sites for both the host and the pathogen, but no significant 478 correlation was observed between the apple and fungus genetic diversity levels across sites 479 (Kendall's tau=0.389, p-value=0.18; Figure S6). Global $F_{S T}$ among sites were 0.259 for apple 480 trees and 0.243 for $V$. inaequalis. Pairwise $F_{S T}$ estimates ranged from 0.000 to 0.207 for apple trees (Table S5) and from 0.000 to 0.721 for $V$. inaequalis (Table S6). For both the host and the pathogen, the orchard (esi_o) was the most differentiated from other sampling sites 483 (Tables S5 and S6). Host and pathogen pairwise differentiation matrices were significantly correlated (Mantel-test Z-score $=0.681, \mathrm{p}$-value $=0.011$; Figure S7). Yet, we found no significant correlation between pairwise $F_{S T}$ and geographical distances matrices, neither for apple trees (Mantel test Z-score= 33.10, p-value=0.42) nor for V. inaequalis (Mantel test Z487 score $=103.20, p$-value $=0.51)$, indicating a lack of isolation by distance . 
489 All pathogen types occur on wild apple trees, but wild-type pathogens occur only on trees with no domesticated ancestry

491 We investigated the association between the fungal pathogen types and the Malus genotypes.

492 From the 240 trees and 255 fungal strains successfully genotyped, we could obtain 249 host-

493 pathogen pairs, several strains having been isolated from the same host trees. A sharp L-

494 shaped pattern was observed when the hybrid index values of the host trees were plotted

495 against those of their associated fungal genotype (Figure 4A). Hybrids were found among

496 both trees and fungi, but no hybrid pathogens were collected from hybrid trees. Hybrid and

497 wild-type fungi were found only on wild apple trees, whereas agricultural-type fungi were

498 found on all types of apple trees, across the whole range of the hybrid index.

\section{Genetic determinants of host adaptation in Venturia inaequalis}

501

502

503

504

505

506

507

508

509

510

In order to identify SNPs predicting the ability of fungal genotypes to parasitize the host species, we performed a DAPC on the genotypes of the $249 \mathrm{~V}$. inaequalis strains, with groups defined a priori based on the assignment of their collection tree as $M$. domestica, hybrid or $M$. sieversii. The first linear discriminant function (LD1) clearly separated the strains sampled on domesticated apple trees from those sampled on wild and hybrid apple trees (Figure S8). The second linear function separated strains found on hybrid apple trees from the ones sampled on M. sieversii (LD2, Figure S8). The analysis of allele contribution (loadings) to the first discriminant function revealed that the SNP V_08160_319 was strongly involved in the genetic differentiation between strains parasitizing $M$. domestica and those found on hybrid and wild hosts (loading of 0.123, Figure S9), while the other SNPs showed much lower 
511 contributions (from $8.723 \times 10^{-8}$ to 0.024 ). The estimate of global $F_{S T}$ was accordingly much

512 higher for the V_08160_319 locus $\left(F_{S T}=0.887\right)$ than for other loci (Figure S10).

513 The SNP V_08160_319 was located in a gene identified as a small secreted protein (SSP) 514 using the protocol described in Le Cam et al. (2019). The two alleles of this SNP were A and

$515 \mathrm{G}$ and corresponded to a non-synonymous site. According to the gene allelic sequences in the 51633 reference genomes, the V_08160_319 SNP was associated to a second one in the same 517 codon, which thus corresponded to a TGA stop codon versus a TAC codon, coding for a 518 tyrosine, at the 140th codon in the 295 amino-acid long protein. Out of the 189 strains for 519 which genotypes for this SNP were available, the stop codon allele indeed had frequencies of 5201.00 on $M$. domestica $(\mathrm{N}=28), 0.92$ on hybrid apple trees $(\mathrm{N}=13)$ and 0.06 on $M$. sieversii 521 (N=148) (Figure 4B). Considering ancestry of fungal strains, the stop codon allele had a 522 frequency of $0.89(\mathrm{~N}=56)$ in the agricultural-type population and 0 in the wild-type population $523(\mathrm{~N}=107)$ and in hybrids $(\mathrm{N}=26)$. The detection of only $\mathrm{A}$ (tyrosine) alleles in hybrids 524 significantly deviated from expectations under the hypothesis of neutral segregation at this 525 locus given the allele frequencies in the pure populations (Binomial-test, $\mathrm{p}$-value $=6.10 \times 10^{-5}$ ).

526 To assess which allele was ancestral, we used five available genomes (Le Cam et al., 2019) 527 belonging to the outgroup V. inaequalis lineage parasitising Pyracantha (Gladieux et al., 528 2010a; Le Cam et al., 2002). These genomes carried the TAC codon, as did the wild-type $V$. 529 inaequalis population on $M$. sieversii. This means that the stop codon truncating the small 530 secreted protein is a derived allele, and has evolved in the agricultural $V$. inaequalis population on M. domestica. 
534 We then investigated experimentally whether the observed association between fungal 535 pathogen types and host tree species resulted from differences in infection ability, by 536 inoculating 57 fungal strains (20 agricultural-type, 17 wild-type and 20 hybrid strains; Table 537 S1) on one genotype of $M$. sieversii and one genotype of $M$. domestica. All agricultural-type 538 strains could cause disease on $M$. domestica $(\mathrm{N}=20$; Figures 5A and 6A), whereas only one 539 wild-type and two hybrid strains could cause disease on $M$. domestica, and even then, only 540 with very low levels of virulence (less than $2 \%$ of diseased leaf area, Figure 5A). All agricultural strains $(\mathrm{N}=20)$, hybrids $(\mathrm{N}=20)$ and wild strains $(\mathrm{N}=17)$ could cause disease on M. sieversii (Figure 5B). Both agricultural-type and hybrid strains were significantly more virulent (i.e. caused larger and/or more lesions) than wild-type strains on $M$. sieversii (Figures 5B and 6B; Wilcoxon tests: $P=1.7 \times 10^{-5}$ and $P=1.2 \times 10^{-4}$, respectively). The $V$. inaequalis 545 hybrids displayed virulence levels on $M$. sieversii that appeared intermediate between the 546 agricultural-type and wild-type populations although the difference between hybrids and the 547 agricultural-type population was not significant (Figure 5B). Similar results were obtained when assignment to species and hybrids were done using the same hybrid index threshold as for apple trees (wild type: Pagr $<0.2$; hybrids: $0.2 \leq$ Pagr $\leq 0.8$; agricultural: Pagr $>0.8$; Figure

550 S11). We found no significant correlation between the proportion of agricultural-type ancestry 551 and the degree of virulence in the hybrid fungal strains (Figure S12; Kendall test: $\tau=-0.047$, $552 P=0.7702)$.

553 The allele at the outlier locus $V \_081690 \_319$ partially explained the virulence on $M$. 554 domestica. Fourty-seven genotypes were available out of 57 strains used in the experiment. 555 Out of the 15 agricultural-type strains with available genotypes, 13 (87\%) carried the stop 556 codon (TGA) allele. Conversely, all the 15 hybrid strains with available genotypes (100\%), as 557 well as all the 17 wild strains (100\%), carried the tyrosine (TAC) allele. When comparing the 
558 two groups of strains corresponding to their alleles at the $V_{-} 081690 \_319$ locus, we found that

559 the strains carrying the stop codon (TGA) allele were more virulent than those carrying the

560 tyrosine (TGA) allele, both on $M$. domestica and on $M$. sieversii (Wilcoxon tests: $P=1.4 \times 10^{-8}$

561 and $P=1.2 \times 10^{-3}$, respectively; Figure S13). Most of the strains carrying the tyrosine allele

562 could not cause disease at all on $M$. domestica, but a few of them could induce a low level of

563 symptoms (Figure S13A).

\section{4}

Demographic inference on the divergence history of wild-type and agricultural-type

\section{Venturia inaequalis populations}

567 We performed demographic inferences in order to test if the observed co-occurrence and 568 hybridization of wild and agricultural-type $V$. inaequalis populations in Kazakhstan resulted 569 from divergence with gene flow or originated from a secondary contact, following the 570 introduction of $M$. domestica, about one century ago in a few orchards in forests of wild $M$.

571 sieversii. We compared the likelihoods of four contrasted scenarios of divergence, with either

572 strict isolation, isolation with continuous migration, isolation with initial migration or 573 secondary contact (Figure 7A).

574 The models allowing gene flow received better support than the strict isolation model (AIC= 575 2403,587014; $\operatorname{LogL}=-1197,793507)$. The model of isolation with migration (IM; AIC= 576 1975,638252; $\log L=-981,8191258)$ did not perform significantly better than the ancient 577 migration model ( $\mathrm{AM} ; \mathrm{AIC}=1977,698045 ; \log \mathrm{L}=-981,8490226)$. The secondary contact 578 model $(\mathrm{SC})$ received the best support $(\mathrm{AIC}=1871,313317, \mathrm{Log} \mathrm{L}=-928,6566583)$, which 579 indicated that the agricultural-type $V$. inaequalis population was likely absent in Asian wild 580 apple forests before the recent introduction of domesticated apple trees in orchards (Figure 581 7B; Table 1). Using the estimate of $\theta$ obtained from the best model (SC, $\theta=143.729$ ), a 
582 mutation rate $\mu$ of $2 \times 10^{-8}$, we found that the ancestral effective size before split, $N_{\text {ref }}$, was near

583 20,000 individuals (Figure 7C). Parameter estimates for the secondary contact model

584 indicated that the divergence between the wild-type and agricultural-type $V$. inaequalis

585 populations occurred 4,570 years ago $(4,570 \pm 324 \mathrm{SD})$ followed by a secondary contact 130

586 years ago (130 $\pm 9 \mathrm{SD})$. This scenario and estimates are consistent with a fungal divergence

587 triggered by apple domestication under strict genetic isolation, followed by a recent secondary

588 contact promoted by modern orchards (Figure 7C).

590 Discussion

591 Gene flow between apple trees species may threaten the genetic integrity of the Asian 592 wild apple

593 Our data show that $M$. sieversii wild apple trees are affected by gene flow following the 594 introduction of the domesticated apple trees in orchards near the natural apple forests of the 595 Kazakh Tian Shan Mountains. Gene flow from M. domestica to M. sieversii had previously 596 been suggested (Cornille et al. 2012; Omasheva et al., 2017), and we reveal here the strong 597 impact of the presence of orchards close to wild forests. Currently, gene flow appears to pose 598 a less severe threat to $M$. sieversii than it does to $M$. sylvestris in Europe, where wild 599 European crabapple tree populations display massive introgression from the cultivated apple 600 tree, to the extent that the European wild species is considered as endangered (Cornille et al., 601 2015; Feurtey et al., 2017). The lower level of introgression in M. sieversii wild populations 602 in Central Asia is probably due to more recent and much less extensive secondary contact 603 between wild and cultivated species than in Europe. The cultivated apple tree was introduced 604 into Europe before the $3^{\text {rd }}$ century BC (Cornille et al., 2014), whereas it was not introduced 
605 into the area sampled in this study until about 100 years ago, and much less extensively than

606 in Europe. Furthermore, the higher density of M. sieversii trees in Kazakh wild forests (Harris

607 et al., 2002; Vavilov, 1931) than of M. sylvestris in European wild forests probably also

608 restricts gene flow from cultivated apple trees. Indeed, pollination distances decrease with

609 density around pollinated trees in European wild apple trees, longer pollination distances

610 increasing the risk of interspecific mating with cultivated trees (Feurtey et al. 2017; Reim et

611 al. 2015).

612 While the overall percentage of apple tree hybrids in the wild forest remained low, near $25 \%$

613 of the trees were found to be hybrids in the forest immediately adjacent to a cultivated

614 orchard, as well as in the forest location that was the closest to the peri-urban environment of

615 Almaty. These findings confirm the weak interspecific reproductive barriers reported for the

616 Malus genus (Zohary \& Hopf, 2000) and raise concerns about the consequences of increasing

617 human-induced changes in this region. As indicated by the IUCN Red List, M. sieversii is

618 already threatened by its range shrinking in Central Asia (Eastwood, Lazkov \& Newton,

619 2009), with a disappearance of more than $70 \%$ of the apple wild forest area over the last 30

620 years due to agricultural expansion and overgrazing. In addition to this range reduction, our

621 results support the view that the genetic integrity of $M$. sieversii may be also threatened. Thus,

622 unless preventive measures are taken to protect Central Asian forests, it may only be a

623 question of time before the genetic integrity of $M$. sieversii deteriorates in Central Asia, as

624 already observed for M. sylvestris in Europe (Cornille et al., 2015; Feurtey et al., 2017), as

625 well as for other European native plant species such as poplars (Bleeker, Schmitz \& Ristow,

626 2007; Rhymer \& Simberloff, 1996; Vanden Broeck, Villar, Van Bockstaele \& Van Slycken,

627 2005). 


\section{Recent invasion of the wild forests by the agricultural $V$. inaequalis population}

630 We confirm in this study the existence of two differentiated $V$. inaequalis populations in

631 Central Asia. The high differentiation level between the agricultural-type and wild-type $V$. 632 inaequalis populations raises the question of whether they should be considered as separate 633 species. However, further studies are needed to assess the taxonomic status of the $V$. 634 inaequalis lineages, especially as hybrids were found.

635 Most importantly, we show that agricultural-type and wild-type $V$. inaequalis populations 636 came into a recent secondary contact after having diverged in strict isolation during apple tree 637 domestication. Indeed, demographic inferences showed that agricultural-type and wild-type fungal populations initially diverged without gene flow about 4,500 years ago, a time consistent with that of apple tree domestication, and came into secondary contact about one century ago, i.e., when domesticated apple trees were introduced in Central Asia. This secondary contact has led to the recent invasion of the agricultural-type population in the wild M. sieversii forest and to the production of hybrids.

The agricultural-type $V$. inaequalis population is able to parasitize both wild and domesticated host trees

646 We uncovered contrasting host range patterns among $V$. inaequalis populations: wild-type and

647 hybrid fungal strains were only found on $M$. sieversii trees, whereas agricultural-type fungal

648 strains were found on trees of all types of ancestry. Pathogenicity tests in controlled 649 conditions matched the distribution observed in nature. Indeed, the agricultural-type fungal 650 strains were able to cause disease on $M$. domestica and M. sieversii whereas the wild-type and 
651 hybrid fungal strains could induce symptoms only on wild host trees. These results, obtained

652 with high spore concentration and in favorable climatic conditions, are conservative and 653 confirm conclusions that were previously obtained with a limited number of $V$. inaequalis 654 strains (Lê Van et al., 2012). Furthermore, we detected agricultural-type V. inaequalis strains 655 at eight different sites in the wild forests on numerous different trees, suggesting that the 656 agricultural-type population can parasitize a large range of $M$. sieversii genotypes. In contrast, 657 no wild-type or hybrid fungal strain was detected on any of the $M$. domestica genotypes present in the orchard. This host range pattern likely explains the significant correlation found between apple tree and $V$. inaequalis pairwise $F_{S T}$ matrices, which was not due to isolation by distance patterns in hosts and pathogens. Indeed, it is likely that the $M$. domestica ancestry proportion in each site drives the allele frequency distribution in $V$. inaequalis by selection, 662 because $M$. domestica can only be parasitized by agricultural-type strains, and not by wild663 type or hybrid pathogens. This situation contrasts with that reported for poplars, in which the hybrid fungal pathogen Melampsora x columbiana can parasitize both pure species and hybrid trees (Newcombe et al., 2000).

In addition, the agricultural $V$. inaequalis population and hybrids displayed greater virulence on wild apple trees than its own endemic wild-type population. This is consistent with the process known as “pestification” (Saleh, Milazzo, Adreit, Fournier \& Tharreau, 2014), under which selection by humans of more resistant plants unwittingly leads pathogens to accumulate more virulences traits and to cause more severe symptoms. This could add further threats for 671 the wild tree species in its natural habitat. Indeed, the scab disease on apple tree leaves reduces photosynthesis (Spotts \& Ferree, 1979), which may affect the growth of the plants.

673 While such increase in virulence may have a limited impact on the growth of adult trees, its 674 effect on the growth of young seedlings could be severe. Furthermore, scab disease can lead 
675 to early fruit fall (MacHardy, 1996), which may decrease seed number, thereby affecting host 676 population dynamics. On $M$. sieversii, hybrid strains were more virulent than wild-type fungal 677 strains but not significantly different from agricultural-type fungal strains. Thus, although the 678 hybridization between fungal lineages does not currently appear to increase damages on wild 679 trees compared to the pure agricultural pathogen, it might participate in the invasion of the 680 disease on the wild host. Future experiments are still needed to estimate precisely the disease 681 fitness effect on seedlings and on the number of seeds produced by adult trees in order to ascertain the long-term impact of this pathogen population on the wild tree natural populations. Nevertheless, our results already suggest that the invasion of the wild forests by the agricultural-type $V$. inaequalis population could further threaten natural $M$. sieversii populations.

\section{The genomic determinants of pestification: a likely new virulence gene}

Our findings suggest the evolution of $V$. inaequalis populations by host tracking of the domesticated apple tree without the loss of its ability to parasitize the wild host. Many resistance genes have been introgressed into $M$. domestica during domestication and modern breeding (Cornille et al., 2012). During its evolutionary tracking of apple trees evolution, the agricultural $V$. inaequalis population has probably accumulated multiple alleles allowing to counteract the crop resistance genes, potentially including the truncated allele of the small secreted protein identified in this study as a putative avirulence gene. Indeed, we found that the host lineage on which $V$. inaequalis strains were sampled was predicted by a SNP in a gene encoding a small secreted protein. In the fungal strains collected on $M$. domestica, the allele corresponded to a stop codon while strains sampled on $M$. sieversii carried the full- 
698

699

700

701

702

703

704

705

706

707

708

709

710

711

712

713

714

715

716

717

718

719

720

length gene. Taken together with the presence of the full-length gene in an outgroup, the $V$. inaequalis lineage parasitizing Pyracantha (Gladieux et al., 2010a; Le Cam et al., 2002), this suggests the acquisition of a virulence allele by the agricultural $V$. inaequalis population during crop host tracking, through the loss of a protein recognized by the domesticated apple tree selected for resistance to apple scab. Such an increase in the virulence of a pathogen following domestication of its host is compatible with the pestification process (Salehet al., 2014), and is consistent with virulence evolution mechanisms previously found in many crop pathogens (Raffaele \& Kamoun, 2012).

The identification of a putative avirulence gene from a small set of 181 SNPs can be explained by the initial choice of these SNPs as the most differentiated between the fungal populations and located within genes. However, we cannot exclude that the V_081690_319 SNP is not directly involved in host adaptation but is instead linked to a genomic determinant of host adaptation. Future functional experiments are required to confirm that the identified gene is an avirulence gene. On the plant side, the identification of the receptor involved in the recognition of this putative avirulent protein could lead to the discovery of a new resistance gene.

More comprehensive studies on whole $V$. inaequalis genomes will likely detect further SNPs involved in host adaptation, as suggested by our data. Indeed, the SNP allele corresponding to the stop codon ( $\mathrm{G}$ allele) was not found in any of the 15 hybrids for which genotypes were available, which significantly deviated from neutral expectations, while agricultural-type strains carrying this allele were found on $M$. sieversii, even if it was at low frequency. In addition, a few strains carrying the full-length allele of the putative avirulence gene were able to cause some symptoms on $M$. domestica in artificial inoculations. This suggests that the stop 
721 codon allele was not selected against on $M$. sieversii in itself but may be deleterious in 722 association with alleles at other loci in hybrids. These results therefore suggest that the locus $723 \quad V \_081690 \_319$ is involved in epistatic interactions with other genes and that hybridization 724 breaks up beneficial allelic combinations required to parasitize $M$. domestica. Furthermore,

725 the V. inaequalis hybrids appeared quantitatively intermediate in virulence on $M$. sieversii, 726 although the virulence difference with the agricultural population was not significant. 727 Intermediate values in hybrids for haploid organisms suggest the implication of multiple 728 genes in virulence.

\section{Conclusion}

731 We found that natural M. sieversii populations are currently only mildly affected by gene flow 732 from $M$. domestica, which was introduced into the area about a century ago but still remains 733 very rare in the Kazakh mountains. Further disturbances in this area might however lead to 734 much higher levels of crop-to-wild gene flow, as already reported in Europe for the European 735 crabapple (Cornille et al., 2013; 2015; Feurtey et al. 2017). In addition, the agricultural-type $736 V$. inaequalis population is invading wild forests and is introgressing the wild-type $V$. 737 inaequalis population, probably due to the greater virulence of the agricultural-type fungal 738 population acquired during its tracking of apple tree domestication. Furthermore, our study 739 represents one of the very rare joint analyses of host and pathogen populations (Croll \& 740 Laine, 2016), despite their importance for understanding the evolutionary mechanisms and

741 histories leading to host specialization and local adaptation. Thanks to the study of host and 742 pathogen pairs, we could identify a putative avirulence gene that may be of great importance 
on a cultivated crop, and a similar approach may be widely used in future studies on host and pathogen populations.

\section{Acknowledgments}

The authors thank C. Peix and kazakh forestry agents for their assistance in sampling, M. Cascales for her assistance in SNPs data analyzing, N. Bierne, R. Nielsen and M. Slatkin for helpful discussions. They acknowledge the staff at the GENTYANE genotyping platform (INRA GDEC, Clermont-Ferrand, France) for SNP and microsatellite genotyping. Authors are also grateful to Pascal Heitzler for providing $M$. sieversii budwoods. They are also most grateful to the PHENOTIC core facility (Angers, France) for its technical support. This study was funded by grants from ANR-12-ADAP-0009 (Gandalf project), from INRA-SPE Department (Escapades project), by a Louis D. grant (Institut de France) to TG, a PhD grant from the Région Ile-de-France to AF, and by the program "Objectif Végétal, Research, Education and Innovation in Pays de la Loire", supported by the French Region Pays de la Loire, Angers Loire Métropole and the European Regional Development Fund (Escapes project).

\section{References}

Bleeker, W., Schmitz, U., \& Ristow, M. (2007). Interspecific hybridisation between alien and native plant species in Germany and its consequences for native biodiversity. Biological Conservation, 137(2), 248-253. doi: 10.1016/j.biocon.2007.02.004

Caffier, V., Lasserre-Zuber, P., Giraud, M., Lascostes, M., Stievenard, R., Lemarquand, A., ... Le Cam, B. (2014). Erosion of quantitative host resistance in the apple $\times$ Venturia inaequalis pathosystem. Infection, Genetics and Evolution, 27, 481-489. doi: 10.1016/j.meegid.2014.02.003 
Christe, C., Stölting, K. N., Paris, M., Fraïsse, C., Bierne, N., \& Lexer, C. (2017). Adaptive evolution and segregating load contribute to the genomic landscape of divergence in two tree species connected by episodic gene flow. Molecular Ecology, 26(1), 59-76. doi: $10.1111 / \mathrm{mec} .13765$

Coffman, A. J., Hsieh, P. H., Gravel, S., \& Gutenkunst, R. N. (2015). Computationally efficient composite likelihood statistics for demographic inference. Molecular Biology and Evolution, 33(2), 591-593. doi: 10.1093/molbev/msv255

Cornille, A., Feurtey, A., Gélin, U., Ropars, J., Misvanderbrugge, K., Gladieux, P., \& Giraud, T. (2015). Anthropogenic and natural drivers of gene flow in a temperate wild fruit tree: a basis for conservation and breeding programs in apples. Evolutionary Applications, 8(4), 373384. doi: 10.1111/eva.12250

Cornille, A., Giraud, T., Smulders, M. J., Roldán-Ruiz, I., \& Gladieux, P. (2014). The domestication and evolutionary ecology of apples. Trends in Genetics, 30(2), 57-65. doi: 10.1016/j.tig.2013.10.002

Cornille, A., Gladieux, P., \& Giraud, T. (2013). Crop-to-wild gene flow and spatial genetic structure in the closest wild relatives of the cultivated apple. Evolutionary Applications, 6(5), 737-748. doi: 10.1111/eva.12059

Cornille, A., Gladieux, P., Smulders, M. J., Roldan-Ruiz, I., Laurens, F., Le Cam, B., ... Gabrielyan, I. (2012). New insight into the history of domesticated apple: secondary contribution of the European wild apple to the genome of cultivated varieties. PLoS genetics, 8(5), e1002703. doi: 10.1371/journal.pgen.1002703

Croll, D., Laine, A. L. (2016). What the population genetic structures of host and pathogen tell us about disease evolution. New Phytologist, 212(3):537-539. doi: 10.1111/nph.14203.

Danecek, P., Auton, A., Abecasis, G., Albers, C. A., Banks, E., DePristo, M. A., ... McVean, G. (2011). The variant call format and VCFtools. Bioinformatics, 27(15), 2156-2158. doi :10.1093/bioinformatics/btr330

Depotter, J. R. L., Seidl, M. F., Wood, T. A., Thomma, B. P. H. J. (2016). Interspecific hybridization impacts host range and pathogenicity of filamentous microbes. Current Opinion in Microbiology, 32:7-13

de Vienne, D. M., Hood, M. E., \& Giraud, T. (2009). Phylogenetic determinants of potential host shifts in fungal pathogens. Journal of Evolutionary Biology, 22(12), 2532-2541. doi: 10.1111/j.1420-9101.2009.01878.x

Dray, S., \& Dufour, A. B. (2007). The ade4 package: implementing the duality diagram for ecologists. Journal of statistical software, 22(4), 1-20. doi: 10.18637/jss.v022.i04

Earl, D. A. \& vonHoldt, B. M. (2012). STRUCTURE HARVESTER: a website and program for visualizing STRUCTURE output and implementing the Evanno method. Conservation Genetics Resources, 4 (2), 359-361. doi: 10.1007/s12686-011-9548-7

Eastwood, A., Lazkov, G., \& Newton, A. (2009). The red list of trees of Central Asia. Fauna and Flora International.

Ellstrand, N. C., Meirmans, P., Rong, J., Bartsch, D., Ghosh, A., De Jong, T. J., ... Strasburg, J. L. (2013). Introgression of crop alleles into wild or weedy populations. Annual Review of 
809 Ecology, Evolution, and Systematics, 44, 325-345. doi: 10.1146/annurev-ecolsys-110512$810 \quad 135840$

811 Evanno, G., Regnaut, S. \& Goudet, J. (2005). Detecting the number of clusters of individuals 812 using the software structure: a simulation study. Molecular Ecology, 14, 2611-2620. 813 doi:10.1111/j.1365-294X.2005.02553.x

814 Falush, D., Stephens, M. \& Pritchard, J. K. (2003). Inference of population structure: 815 Extensions to linked loci and correlated allele frequencies. Genetics, 164(8), 1567-1587.

816 Feurtey, A., Cornille, A., Shykoff, J. A., Snirc, A., \& Giraud, T. (2017). Crop-to-wild gene 817 flow and its fitness consequences for a wild fruit tree: Towards a comprehensive conservation 818 strategy of the wild apple in Europe. Evolutionary Applications, 10(2), 180-188. doi: $819 \quad 10.1111 /$ eva.12441

820 Feurtey, A., \& Stukenbrock, E. H. (2018). Interspecific gene exchange as a driver of adaptive 821 evolution in fungi. Annual Review of Microbiology, 72, 377-398. doi: 10.1146/annurev-micro$822 \quad 090817-062753$

823 Forsline, P. L., Aldwinckle, H. S., Dickson, E. E., Luby, J. J., \& Hokanson, S. C. (2003). 824 Collection, maintenance, characterization, and utilization of wild apples of Central Asia. 825 Horticultural reviews, 29, 1-62.

826 Giraud, T., Refrégier, G., Le Gac, M., de Vienne, D. M., \& Hood, M. E. (2008). Speciation in 827 fungi. Fungal Genetics and Biology, 45(6), 791-802. doi: 10.1016/j.fgb.2008.02.001

828 Gladieux, P., Caffier, V., Devaux, M., Le Cam, B. (2010a). Host-specific differentiation 829 among populations of Venturia inaequalis causing scab on apple, pyracantha and loquat. 830 Fungal Genetics and Biology, 47(6), 511-521. doi: 10.1016/j.fgb.2009.12.007

831 Gladieux, P., Vercken, E., Fontaine, M. C., Hood, M. E., Jonot, O., Couloux, A., \& Giraud, T. 832 (2011). Maintenance of fungal pathogen species that are specialized to different hosts: 833 allopatric divergence and introgression through secondary contact. Molecular Biology and 834 Evolution, 28(1), 459-471. doi: 10.1093/molbev/msq235

835 Gladieux, P., Zhang, X. G., Afoufa-Bastien, D., Sanhueza, R. M. V., Sbaghi, M., \& Le Cam, 836 B. (2008). On the origin and spread of the scab disease of apple: out of central Asia. PLoS 837 One, 3(1), e1455. doi: 10.1371/journal.pone.0001455

838 Gladieux, P., Zhang, X. G., RóLdan-Ruiz, I., Caffier, V., Leroy, T., Devaux, M., ... Le Cam, 839 B. (2010b). Evolution of the population structure of Venturia inaequalis, the apple scab 840 fungus, associated with the domestication of its host. Molecular Ecology, 19(4), 658-674. doi $841 \quad: 10.1111 /$ j.1365-294X.2009.04498.x

842 Gompert, Z., \& Alex Buerkle, C. (2010). INTROGRESS: a software package for mapping 843 components of isolation in hybrids. Molecular Ecology Resources, 10(2), 378-384. doi: $844 \quad 10.1111 / \mathrm{j} .1755-0998.2009 .02733 . x$

845 Gutenkunst, R. N., Hernandez, R. D., Williamson, S. H., \& Bustamante, C. D. (2009). 846 Inferring the joint demographic history of multiple populations from multidimensional SNP 847 frequency data. PLoS Genetics, 5(10), e1000695. doi: 10.1371/journal.pgen.1000695 
848 Hanage, W. P., Fraser, C., Tang, J., Connor, T. R., \& Corander, J. (2009). Hyper849 recombination, diversity, and antibiotic resistance in pneumococcus. Science, 324(5933), 850 1454-1457. doi: 10.1126/science. 1171908

851 Harris, S. A., Robinson, J. P., \& Juniper, B. E. (2002). Genetic clues to the origin of the apple. 852 Trends in Genetics, 18(8), 426-430. doi: 10.1016/S0168-9525(02)02689-6

853 Harshman, J. M., Evans, K. M., Allen, H., Potts, R., Flamenco, J., Aldwinckle, H. S., ... 854 Norelli, J. L. (2017). Fire blight resistance in wild accessions of Malus sieversii. Plant 855 disease, 101(10), 1738-1745. doi: 10.1094/PDIS-01-17-0077-RE

856 Hooftman, D. A. P., Jong, M. J. D., Oostermeijer, J. G. B., \& Den Nijs, H. J. C. M. (2007). 857 Modelling the long-term consequences of crop-wild relative hybridization: a case study using 858 four generations of hybrids. Journal of Applied Ecology, 44(5), 1035-1045. doi: 859 10.1111/j.1365-2664.2007.01341.x

860 Hovick, S. M., Campbell, L. G., Snow, A. A., \& Whitney, K. D. (2012). Hybridization alters 861 early life-history traits and increases plant colonization success in a novel region. The 862 American Naturalist, 179(2), 192-203. doi: 10.1086/663684

863 Ioos, R., Andrieux, A., Marçais, B., \& Frey, P. (2006). Genetic characterization of the natural 864 hybrid species Phytophthora alni as inferred from nuclear and mitochondrial DNA analyses. 865 Fungal Genetics and Biology, 43(7), 511-529. doi: 10.1016/j.fgb.2006.02.006

866 Jakobsson, M. \& Rosenberg, N. A. (2007). CLUMPP: a cluster matching and permutation 867 program for dealing with label switching and multimodality in analysis of population 868 structure. Bioinformatics, 23(14), 1801-1806.

869 Jombart, T. (2008). adegenet: a R package for the multivariate analysis of genetic markers. 870 Bioinformatics, 24, 1403-1405. doi: 10.1093/bioinformatics/btn129

871 Jombart, T., Devillard, S., \& Balloux, F. (2010). Discriminant analysis of principal 872 components: a new method for the analysis of genetically structured populations. $B M C$ 873 Genetics 11: 94.

874 Le Cam, B., Parisi, L. \& Arene, L. (2002). Evidence of two formae speciales in Venturia 875 inaequalis, responsible for apple and Pyracantha scab. Phytopathology, 92, 314-320.

876 Le Cam, B., Sargent, D., Gouzy, J., Amselem, J., Bellanger, M. N., Bouchez, O., ... Lemaire, 877 C. (2019). Population genome sequencing of the scab fungal species Venturia inaequalis, 878 Venturia pirina, Venturia aucupariae and Venturia asperata. G3: Genes, Genomes, Genetics, 879 9(8), 2405-2414. doi: 10.1534/g3.119.400047

880 Le Gac, M. L., \& Giraud, T. (2008). Existence of a pattern of reproductive character 881 displacement in Homobasidiomycota but not in Ascomycota. Journal of evolutionary biology, 882 21(3), 761-772. doi: 10.1111/j.1420-9101.2008.01511.x

883 Lê Van, A., Gladieux, P., Lemaire, C., Cornille, A., Giraud, T., Durel, C. E., ... Le Cam, B. 884 (2012). Evolution of pathogenicity traits in the apple scab fungal pathogen in response to the 885 domestication of its host. Evolutionary applications, 5(7), 694-704. doi: 10.1111/j.1752$886 \quad 4571.2012 .00246 . x$

887 Lemaire, C., De Gracia, M., Leroy, T., Michalecka, M., Lindhard-Pedersen, H., Guerin, F., ... 888 Le Cam, B. (2016). Emergence of new virulent populations of apple scab from 
889 nonagricultural disease reservoirs. New Phytologist, 209(3), 1220-1229. doi: $890 \quad 10.1111 /$ nph.13658

891 Leroy, T., Caffier, V., Celton, J. M., Anger, N., Durel, C. E., Lemaire, C., \& Le Cam, B. 892 (2016). When virulence originates from nonagricultural hosts: evolutionary and 893 epidemiological consequences of introgressions following secondary contacts in Venturia 894 inaequalis. New Phytologist, 210(4), 1443-1452. doi: 10.1111/nph.13873

895 Leroy, T., Lemaire, C., Dunemann, F., \& Le Cam, B. (2013). The genetic structure of a 896 Venturia inaequalis population in a heterogeneous host population composed of different 897 Malus species. BMC evolutionary biology, 13(1), 64. doi: 10.1186/1471-2148-13-64

898

899

900

901

902

903

904

905

906

907

908

909

910

911

912

913

914

915

916

917

918

919

920

921

922

923

924

925

926

927

Lin, X., Litvintseva, A. P., Nielsen, K., Patel, S., Floyd, A., Mitchell, T. G., \& Heitman, J. (2007). $\alpha \mathrm{AD} \alpha$ hybrids of Cryptococcus neoformans: evidence of same-sex mating in nature and hybrid fitness. PLoS Genetics, 3(10), e186. doi: 10.1371/journal.pgen.0030186

Liu, B. H., Cheng, L., Ma, F. W., Liang, D., \& Zou, Y. J. (2012). Influence of rootstock on drought response in young 'Gale Gala' apple (Malus domestica Borkh.) trees. Journal of the Science of Food and Agriculture, 92(12), 2421-2427. doi: 10.1002/jsfa.5647

MacHardy, W. E. (1996). Apple scab: biology, epidemiology, and management. American Phytopathological Society (APS Press).

Mantel, N. (1967). The detection of disease clustering and a generalized regression approach. Cancer Research 27, 209-20.

Newcombe, G., Stirling, B., McDonald, S., \& Bradshaw, H. D. (2000). Melampsorax columbiana, a natural hybrid of M. medusae and M. occidentalis. Mycological Research, 104(3), 261-274. doi: 10.1017/S0953756299001665

Nikiforova, S. V., Cavalieri, D., Velasco, R., \& Goremykin, V. (2013). Phylogenetic analysis of 47 chloroplast genomes clarifies the contribution of wild species to the domesticated apple maternal line. Molecular Biology and Evolution, 30(8), 1751-1760. doi: 10.1093/molbev/mst092

Omasheva, M. Y., Flachowsky, H., Ryabushkina, N. A., Pozharskiy, A. S., Galiakparov, N. N., \& Hanke, M. V. (2017). To what extent do wild apples in Kazakhstan retain their genetic integrity? Tree genetics \& genomes, 13(3), 52. doi: 10.1007/s11295-017-1134-z

Paradis, E. \& Schliep, K. (2019). ape 5.0: an environment for modern phylogenetics and evolutionary analyses in R, Bioinformatics, 35(3), 526-528. https://doi.org/10.1093/bioinformatics/bty633

Raffaele, S., Kamoun, S. (2012). Genome evolution in filamentous plant pathogens: why bigger can be better. Nature Reviews Microbiology 10, 417-430. doi: 10.1038/nrmicro2790

R Core Team (2018). R: A language and environment for statistical computing. R Foundation for Statistical Computing, Vienna. URL: http://www.R-project.org/

Reim, S., Proft, A., Heinz, S., Lochschmidt, F., Höfer, M., Tröber, U., \& Wolf, H. (2017). Pollen movement in a Malus sylvestris population and conclusions for conservation measures. Plant Genetic Resources, 15(1), 12-20. doi:10.1017/S1479262115000301 
928 Rhymer, J. M., \& Simberloff, D. (1996). Extinction by hybridization and introgression.

929 Annual Review of Ecology and Systematics, 27(1), 83-109. doi:

930 10.1146/annurev.ecolsys.27.1.83

931 Rousset, F. (2008). Genepop'007: a complete re $\square$ implementation of the genepop software for 932 Windows and Linux. Molecular Ecology Resources, 8, 103-106. doi:10.1111/j.1471933 8286.2007.01931.x

934 Saleh, D., Milazzo, J., Adreit, H., Fournier, E., \& Tharreau, D. (2014). South-East Asia is the 935 center of origin, diversity and dispersion of the rice blast fungus, Magnaporthe oryzae. New 936 Phytologist, 201(4), 1440-1456. doi: 10.1111/nph.12627

937 Spotts, R. A., \& Ferree, D.C. (1979). Photosynthesis, transpiration, and water potential of 938 apple leaves infected by Venturia inaequalis. Phytopathology, 69(7), 717-719. doi: 939 10.1094/Phyto-69-717

940 Stukenbrock, E. H., Christiansen, F. B., Hansen, T. T., Dutheil, J. Y., \& Schierup, M. H. 941 (2012). Fusion of two divergent fungal individuals led to the recent emergence of a unique 942 widespread pathogen species. Proceedings of the National Academy of Sciences, 109(27), 943 10954-10959. doi: 10.1073/pnas.1201403109

944 Stukenbrock, E. H. (2016a). Hybridization speeds up the emergence and evolution of a new 945 pathogen species. Nature genetics, 48(2), 113. doi: 10.1038/ng.3494

946 Stukenbrock, E. H. (2016b). The role of hybridization in the evolution and emergence of new 947 fungal plant pathogens. Phytopathology, 106(2), 104-112. doi: 10.1094/PHYTO-08-15-0184948 RVW

949 Tine, M., Kuhl, H., Gagnaire, P. A., Louro, B., Desmarais, E., Martins, R. S., ... Dieterich, R. 950 (2014). European sea bass genome and its variation provide insights into adaptation to 951 euryhalinity and speciation. Nature communications, 5, 5770. doi: 10.1038/ncomms6770

952 Todesco, M., Pascual, M. A., Owens, G. L., Ostevik, K. L., Moyers, B. T., Hübner, S., ... 953 Rieseberg, L. H. (2016). Hybridization and extinction. Evolutionary Applications, 9(7), 892954 908. doi: 10.1111/eva.12367

955 Vanden Broeck, A. V., Villar, M., Van Bockstaele, E., \& Van Slycken, J. (2005). Natural 956 hybridization between cultivated poplars and their wild relatives: evidence and consequences 957 for native poplar populations. Annals of Forest Science, 62(7), 601-613. doi: 958 10.1051/forest:2005072

959 Vavilov NI. (1931). The role of Central Asia in the origin of cultivated plants. Bulletin of 960 Applied Botany Genetics and Plant Breeding 26(3), 3-44.

961 Velasco, R., Zharkikh, A., Affourtit, J., Dhingra, A., Cestaro, A., Kalyanaraman, A., ... Salvi, 962 S. (2010). The genome of the domesticated apple (Malus $\times$ domestica Borkh.). Nature 963 genetics, 42(10), 833. doi: 10.1038/ng.654

964 Wang, H., Vieira, F.G., Crawford, J. E., Chu, C. \& Nielsen, R. (2017).Asian wild rice is a 965 hybrid swarm with extensive gene flow and feralization from domesticated rice. Genome 966 Research,27(6),1029-1038. doi: 10.1101/gr.204800.116. 
967 Wang, Y., Arenas, C. D., Stoebel, D. M., Flynn, K., Knapp, E., Dillon, M. M., ... Cooper, T. 968 F. (2016). Benefit of transferred mutations is better predicted by the fitness of recipients than 969 by their ecological or genetic relatedness. Proceedings of the National Academy of Sciences, 970 113(18), 5047-5052. doi: 10.1073/pnas.1524988113

971 Weir, B. S., \& Cockerham, C. C. 1984. Estimating F-statistics for the analysis of population 972 structure. Evolution 38: 1358-70.

973 Wolf, D. E., Takebayashi, N., \& Rieseberg, L. H. (2001). Predicting the risk of extinction 974 through hybridization. Conservation Biology, 15(4), 1039-1053. doi: 10.1046/j.1523975 1739.2001.0150041039.x

976 Zeder, M. A., Emshwiller, E., Smith, B. D., \& Bradley, D. G. (2006). Documenting 977 domestication: the intersection of genetics and archaeology. Trends in Genetics, 22(3), 139978 155. doi: 10.1016/j.tig.2006.01.007

979 Zohary, D., \& Hopf, M. (2000). Domestication of plants in the Old World: the origin and 980 spread of cultivated plants in West Asia, Europe, and the Nile Valley. Oxford University 981 Press, New York.

982

983

984

\section{Data accessibility}

985

All data will be archived and available on the following link, doi: 10.5281/zenodo.3565732

\section{Author Contributions}

989

990

991

992

993

994

995

996

997

998

999

1000

1001
AF, EG, TG and CL wrote the manuscript with inputs from BLC, VC, JS and LD. Sampling in Kazakhstan was performed by CL and BLC. Apple microsatellite genotyping was performed by AF. Apple scab SNP design and genotyping was performed by MDG, MS and CL. SNP mapping and calling was performed by LD and JS. EG, MNB, PE and VC managed in vitro cultures of Venturia inaequalis and pathology assays. AF, EG and CL performed population genetics and statistical analyses. CL, BLC and TG conceived the project and obtained financial support. 
bioRxiv preprint doi: https://doi.org/10.1101/2020.04.15.042242; this version posted April 16, 2020. The copyright holder for this preprint (which was not certified by peer review) is the author/funder. All rights reserved. No reuse allowed without permission.

1002

1003

1004 
1005

1006

1007

1008

1009

1010

1011

1012

1013

1014

1015

1016

1017

1018

1019

1020

1021

1022

1023

1024

1025

\section{Tables}

Table 1: Comparison of four alternative demographic models of divergence between the reference wild-type and agricultural-type populations of Venturia inaequalis using $\partial \mathrm{a} \partial \mathrm{i}$ on the SNP dataset.

\begin{tabular}{|c|c|c|c|c|c|c|c|c|c|c|c|}
\hline Model & $k$ & $\log \mathrm{L}$ & $\mathrm{AIC}$ & $\begin{array}{c}\text { Model } \\
\text { Compar } \\
\text { ison }\end{array}$ & $\mathrm{N}_{\mathrm{Wild}}$ & $\mathrm{N}_{\text {Agri }}$ & $\mathrm{M}_{\mathrm{WA}}$ & $\mathrm{M}_{\mathrm{AW}}$ & $\mathrm{T}_{\mathrm{S}}$ & $\mathrm{T}_{\mathrm{AM}}$ & $\mathrm{T}_{\mathrm{SC}}$ \\
\hline $\mathrm{SI}$ & 3 & - & 2403.587014 & - & 0.094 & 1.360 & - & - & 0.092 & - & - \\
\hline $\mathrm{IM}$ & 5 & -981.8 & 1975.638252 & $\mathrm{SI}^{* * *}$ & 0.139 & 0.898 & 1.259 & 1.131 & 0.460 & - & - \\
\hline AM & 6 & -981.8 & 1977.698045 & $\begin{array}{c}\mathrm{SI}^{* * *} \\
\mathrm{IM}^{\mathrm{NS}}\end{array}$ & 0.139 & 0.892 & 1.270 & 1.140 & 0.460 & $1.09 \times 10^{-9}$ & - \\
\hline SC & $\mathbf{6}$ & $\mathbf{- 9 2 8 . 6}$ & $\mathbf{1 8 7 1 . 3 1 3 3 1 7}$ & $\begin{array}{c}\mathbf{S I}^{* * *}, \\
\mathbf{I M}^{* * *}, \\
\mathbf{A M}^{+++}\end{array}$ & $\mathbf{0 . 1 6 3}$ & $\mathbf{0 . 9 9 1}$ & $\mathbf{1 . 3 5 5}$ & $\mathbf{4 . 3 8 9}$ & $\mathbf{0 . 2 2 7}$ & $\mathbf{-}$ & $\mathbf{0 . 0 0 6}$ \\
\hline
\end{tabular}

SI, strict isolation model; IM, isolation with migration model; AM, ancient migration model; SC, secondary contact model; $k$, parameter numbers; $\operatorname{logL}$, log-likelihood; AIC, Akaike Information Criterion; $\mathrm{N}_{\text {Wild }}$, effective sizes of wild-type lineage; $\mathrm{N}_{\mathrm{Agri}}$, effective sizes of agricultural-type lineage; $M_{W A}$, migration rates from wild to agricultural-type lineage; $M_{A W}$, migration rates from agricultural-type to wild-type lineage; $\mathrm{T}_{\mathrm{S}}$, time of split; $\mathrm{T}_{\mathrm{AM}}$, duration time since ancient migration; $\mathrm{T}_{\mathrm{SC}}$, duration time since secondary contact.

The best supported model (SC) is indicated in bold. Model comparisons within and between classes of models are shown. Nested models were compared using likelihood ratio tests, with subscripts indicating significance levels (abbreviated $* * * \mathrm{P}<0.001 ; * * \mathrm{P}<0.01 ; * \mathrm{P}<0.05, \mathrm{NS}$ non-significant). Non-nested models were compared using AIC with relative likelihood of each model compared to the best model $\mathrm{L}(\mathrm{Mi} \mid \mathrm{Mbest})=\exp ((\mathrm{AICmin}-\mathrm{AICi}) / 2)$ (abbreviated $+++\mathrm{L}($ Mi|Mbest $)<0.001 ;++\mathrm{L}($ Mi|Mbest $)<0.01 ;+\mathrm{L}($ Mi $\mid$ Mbest $)<0.05, \mathrm{~L}($ Mi|Mbest $)>0.05$ : AIC difference shown). 


\section{Figure legends}

1028 Figure 1: Summary of previous studies and questions addressed in the current study on Malus 1029 apple trees (A) and on their fungal pathogen Venturia inaequalis (B). A. The divergence and gene flow are shown at the top between the domesticated apple tree $M$. domestica, its initial progenitor M. sieversii from Asia and its secondary contributor the European crabapple tree M. sylvestris (Cornille et al. 2012). The distribution areas of the two wild apple trees are shown at the bottom, indicating (1) the initial domestication in Asia (Cornille et al. 2012), migration along the Silk Road (yellow full line) and (2) secondary gene flow between $M$. domestica and M. sylvestris in Europe (Cornille et al. 2013; Feurtey et al. 2017), followed by dispersion of modern cultivars worldwide (dashed yellow arrows). (3) A couple of previous studies have investigated the consequence of the secondary contact between the domesticated apple and its Asian progenitor in terms of gene flow from $M$. domestica to $M$. sieversii (Cornille et al. 2012; 2012; Omasheva et al., 2017), and here we study the consequences of planting orchards in forest. B. The divergence is shown at the top between the fungal pathogen $V$. inaequalis populations and their distribution area at the bottom, with colored trees symbolizing apple tree species and colored circles the fungal populations. (1) Gladieux et al. (2010b) showed the existence of differentiated populations in Europe on M. silvestris and $M$. domestica (1A), in anthropic Central Asian areas on M. sieversii and M. domestica (1B) and in the wild Central Asian forests on M. sieversii (1C). (2) Lemaire et al. (2016) showed that the differentiated European $V$. inaequalis population on cultivated apples carrying the Rvi6 resistance originated from M. floribunda. (3) Leroy et al. (2016) investigated the existence of gene flow between populations infecting Riv6 and non-Riv6 apple trees in Europe. (4) The present study investigates the occurrence of gene flow among Central Asian pathogen populations, i.e., the population in anthropic Central Asian areas on $M$. sieversii and $M$. domestica and the population in the wild Central Asian forests on $M$. sieversii.

1053

Figure 2: Genetic diversities and ancestry assignments for apple trees and Venturia inaequalis. (A) Principal component analysis on genotypes of 240 apple trees (Malus spp.) for 28 microsatellite markers, with their ancestry assignment $\left(P_{d o m}\right)$ to the cultivated apple tree $M$. domestica indicated by color. The distribution of the $P_{d o m}$ index is shown in the inset at the top left. (B) Principal component analysis on genotypes of $255 \mathrm{~V}$. inaequalis strains for 181 SNPs, with their ancestry assignment $\left(P_{a g r}\right)$ to the agricultural-type of $V$. inaequalis indicated by color. The distribution of the $P_{a g r}$ index is shown in the inset at the top left.

1061

Figure 3: Distribution of the samples of apple trees and their fungal pathogen Venturia inaequalis in nine sites in the Tian Shan Mountains near Almaty (Kazakhstan). The black dot on the upper left corner map represents the global sampling location. Proportions of wild (blue), domesticated/agricultural (yellow) and hybrid (red) genotypes are shown for each sampling site for Malus trees (upper row of pie charts) and for their scab fungal pathogen (lower row of pie charts). The pie charts below the figure correspond to sampling sites in the natural forest. The pie charts above the figure refer to a sampling location composed of two sites, an orchard and the natural forest immediately surrounding it. Codes of the sampling 
1070 locations: kot at Koturbulac; tag and tar at Talghar; lac, gec and esi (esi_o for the orchard and esi_f for the surrounding forest) at Esik; tuk and tum at Turgen.

1072

Figure 4: The proportion of Malus domestica ancestry in the apple tree ( $y$ axis) plotted against the proportion of agricultural-type ancestry in Venturia inaequalis strains (x axis). A) For each of the 249 host-pathogen pairs, two sampling sites are highlighted: the orchard (esi_o) in orange and the surrounding forest (esi_f) in blue. B) For each of the 189 hostpathogen pairs for which the genotype at the $V \_081690 \_319$ locus was available, the $V$. inaequalis strains carrying the tyrosine (A) allele were colored in grey and the ones carrying the stop codon $(\mathrm{G})$ allele in red.

Figure 5: Results of pathogenicity experiments in controlled conditions consisting in artificial inoculation of the fungus Venturia inaequalis (57 fungal strains; mean of three replicates per strain, one genotype per apple species) on apple tree leaves (Malus sieversii and $M$. domestica). Boxplots of the percentage of scabbed leaf area for wild-type, agricultural-type and hybrid $V$. inaequalis strains of A) $M$. domestica at 21 days post inoculation (dpi) and B) $M$. sieversii at $19 \mathrm{dpi}$. The box represents the lower and the upper quartiles. The thick horizontal line represents the median. The whiskers represent the largest and lowest observed values that fall within the distance of 1.5 times the interquartile range. The points represent the mean values of the percentage of diseased leaf area for each strain across the three replicates. Different letters indicate significant differences between populations $(P<0.05$; Wilcoxon's rank sum tests).

Figure 6: Pictures illustrating the percentage of scabbed leaf area for the most virulent wildtype, agricultural-type and hybrid Venturia inaequalis strains on A) Malus domestica at 21 days post inoculation (dpi) and B) M. sieversii at 19 dpi.

1096

Figure 7: Demographic inference on the divergence between wild and agricultural types of Venturia inaequalis in Kazakhstan. A. The four contrasted scenarios of divergence compared, with either strict isolation (SI), isolation with continuous migration (IM), isolation with initial migration (AM) and secondary contact (SC), with effective populations sizes of the ancestral population $\left(\mathrm{N}_{\mathrm{ref}}\right)$ and of the two daughter populations $\left(\mathrm{N}_{\mathrm{w}}\right.$ and $\left.\mathrm{N}_{\mathrm{A}}\right)$, the rate of gene flow in the two directions $\left(\mathrm{M}_{\mathrm{W}}\right.$ and $\left.\mathrm{M}_{\mathrm{A}}\right)$ and the time since the cessation of gene flow $\left(\mathrm{T}_{\mathrm{AM}}\right)$ or the secondary contact $\left(\mathrm{T}_{\mathrm{SC}}\right)$. B. The joint allele-frequency spectrum (AFS) for the wild and agricultural fungal populations, showing the count of derived allele. Each entry of the joint AFS is colored by the number of SNPs in it, according to the scale shown. C. The secondary 1106 contact (SC) model that obtained the best support and estimates of the parameters. 


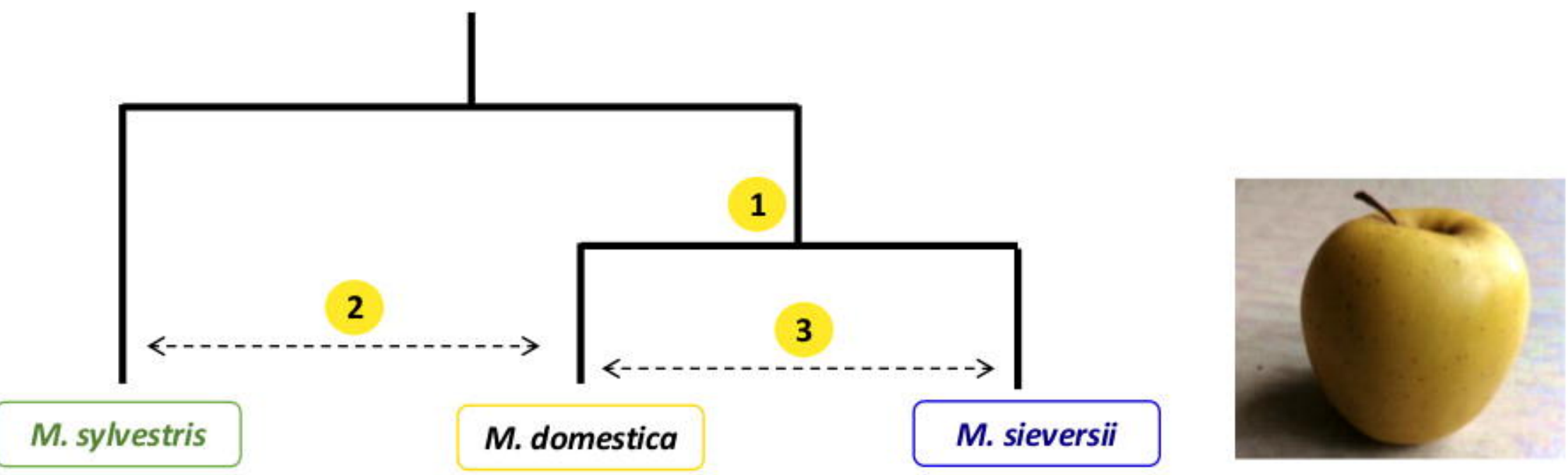

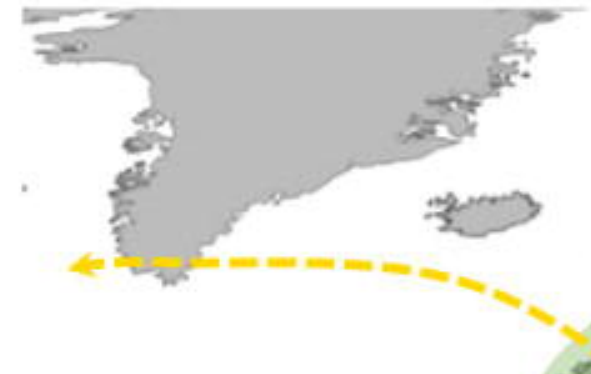

M. sylvestris

:

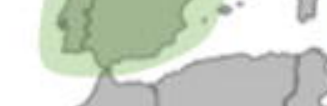

2 Dissemination and hybridization with the European M. sylvestris crabapple. Severe impact of gene flow on wild crabapples.

(Cornille et al. 2013 \& 2015 ; Feurtey et al. 2017)
3 Secondary contact and gene flow from $M$. domestica to $M$. sieversii (Cornille et al. 2012; Omasheva et al., 2017)

4 Impact of secondary contact on the natural forests in the Tian Shan mountains? (This study) 
2 The European V. inaequalis population on cultivated apples carrying the Rvi6 resistance originated from $M$. floribunda (Lemaire et al. 2016)
1 Identification of $3 \mathrm{~V}$. inaequalis populations (Gladieux et al 2010b):

- $1 \mathrm{~A}$ in Europe

- $1 \mathrm{~B}$ in anthropic Central Asian areas

- $1 \mathrm{C}$ in the wild Central Asian forests

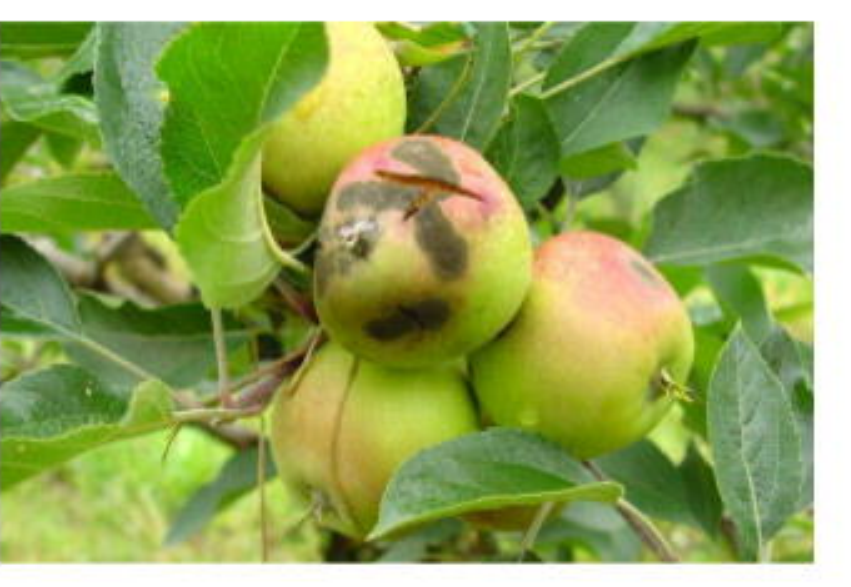

3

Venturia inaequalis

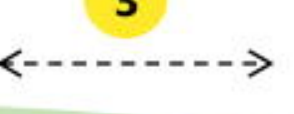

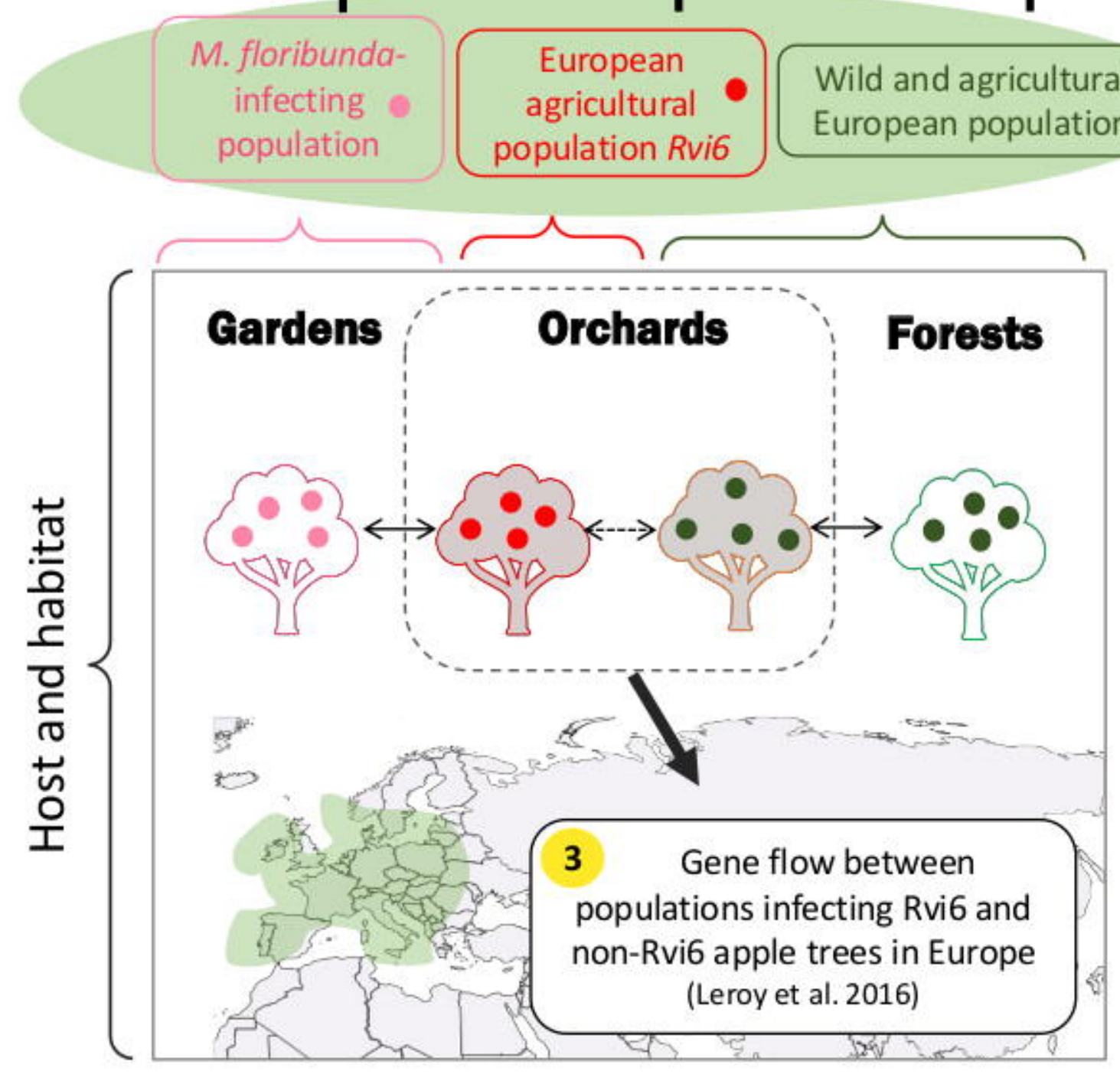

\section{1}

4 $<-\cdots-\cdots$
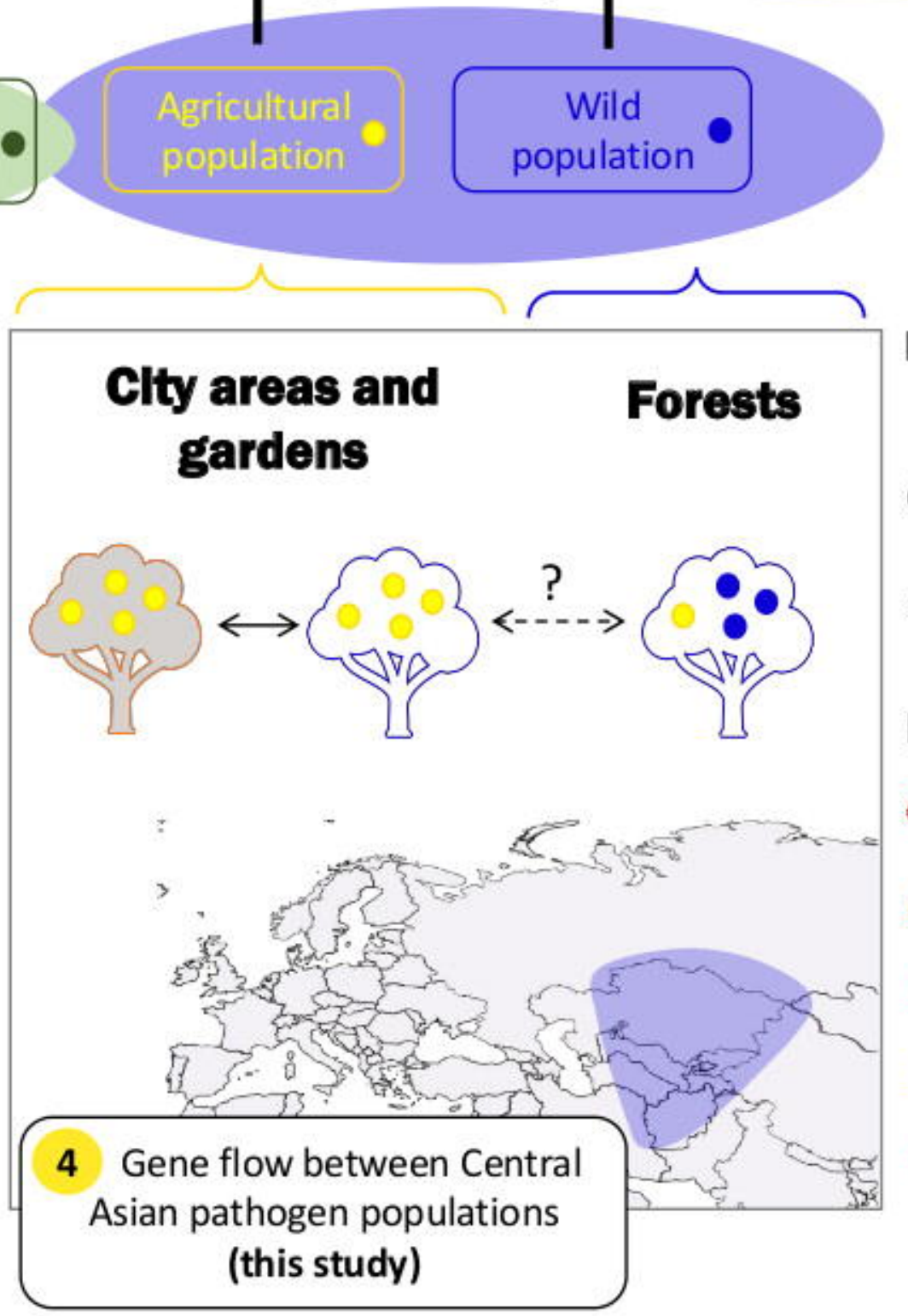

Legend shapes:

- $V$. inaequalis

wipe Wild apple tree

Domesticated apple tree

\section{Legend host color:}

M. domestica with Rvi6

M. domestica non-Rvi6

M. sylvestris

M. sieversii

M. floribunda 


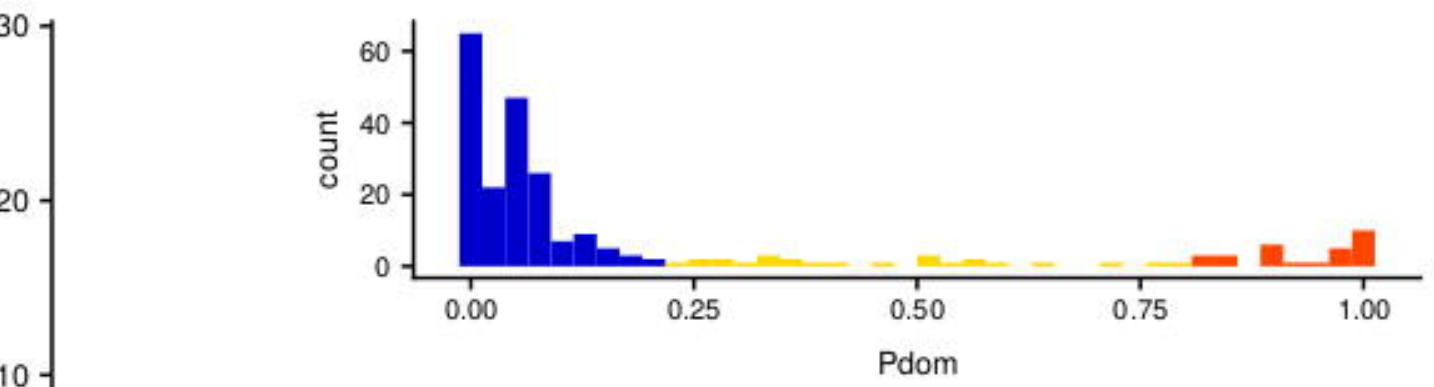

•.0.

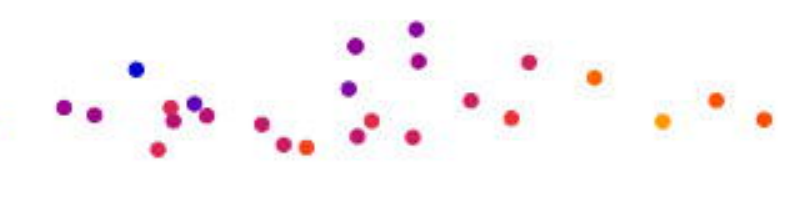

$-5$

Principal component 1 (6\%)

B

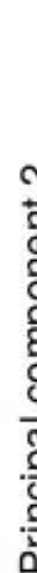

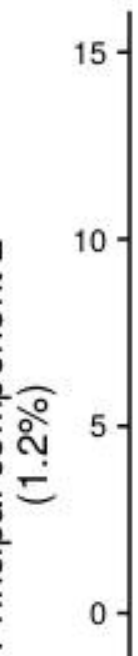

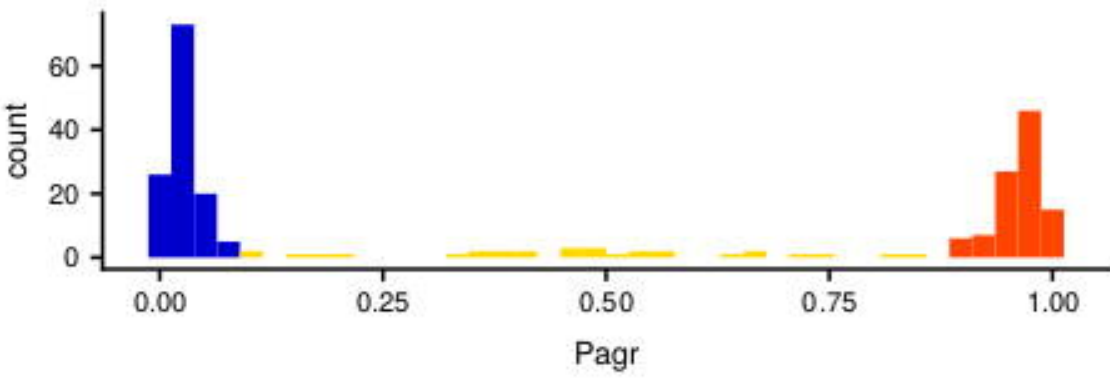

ann-

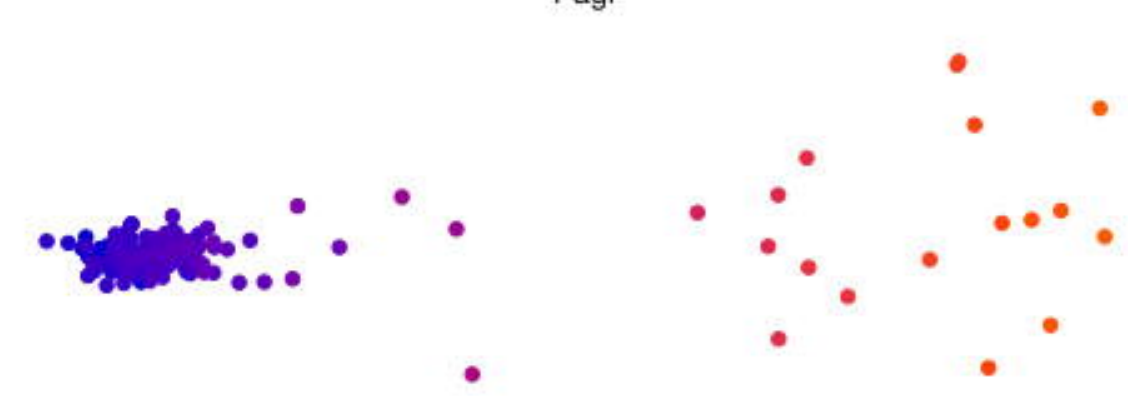

Pagr

1.00
-0.75
-0.50
-0.25
0.00
Pdom

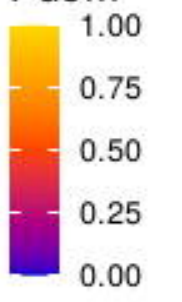



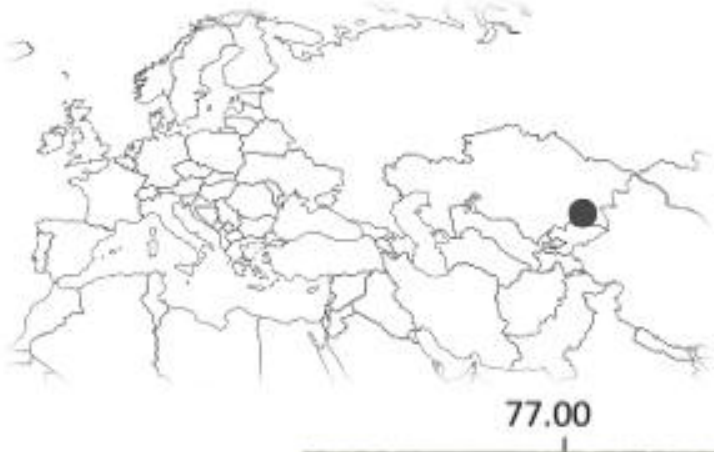

orchard surrounding forest

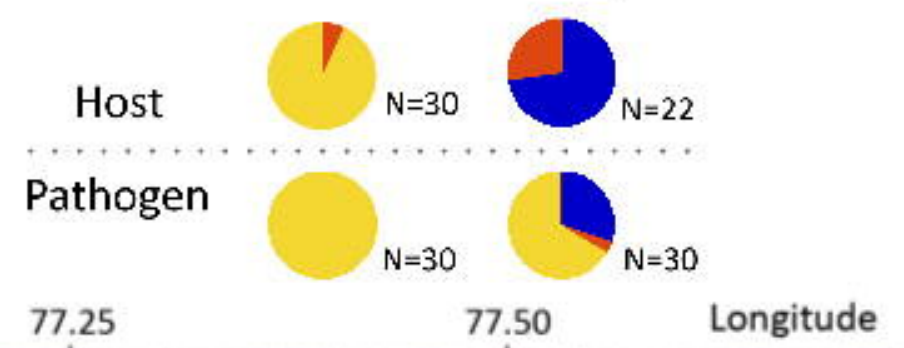

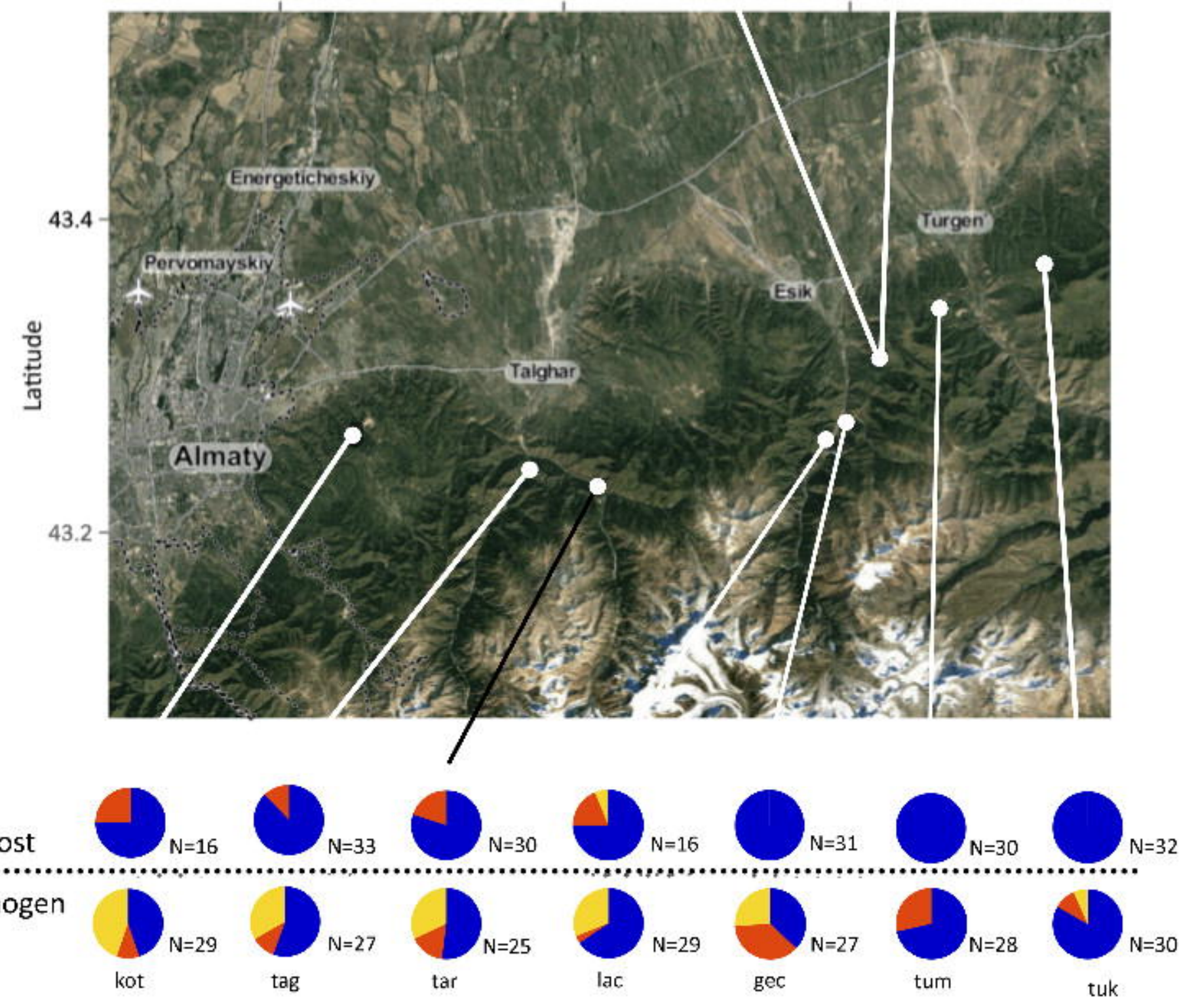




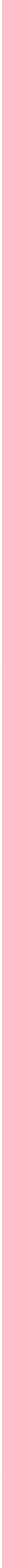


A
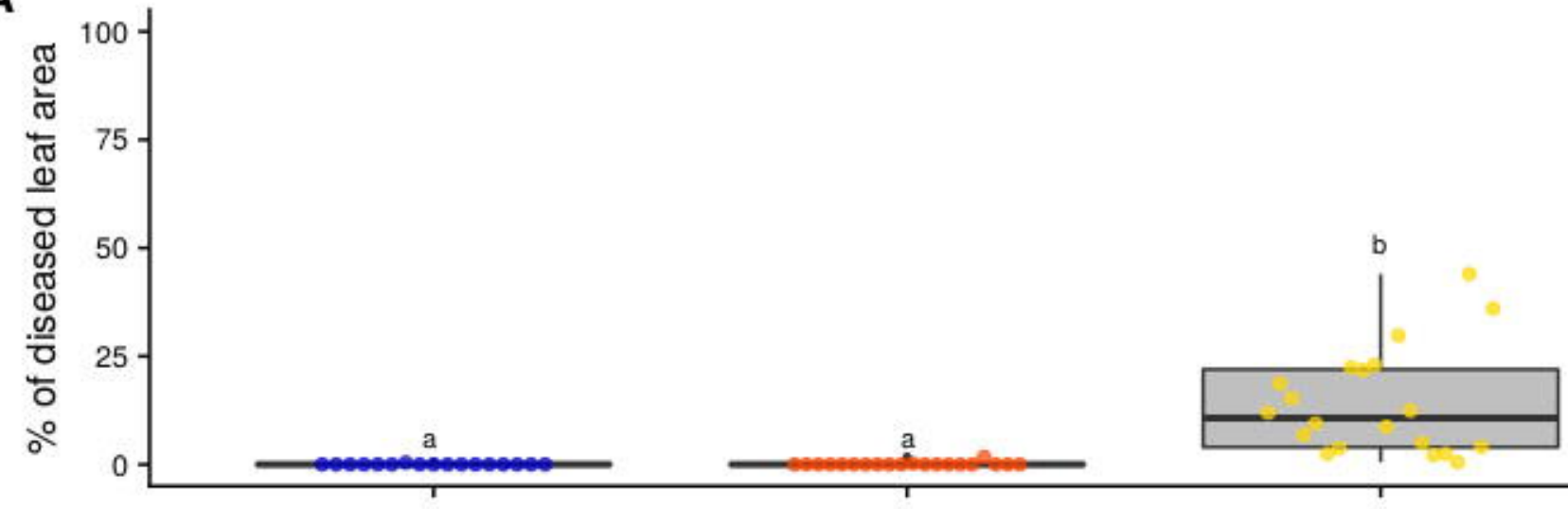

B
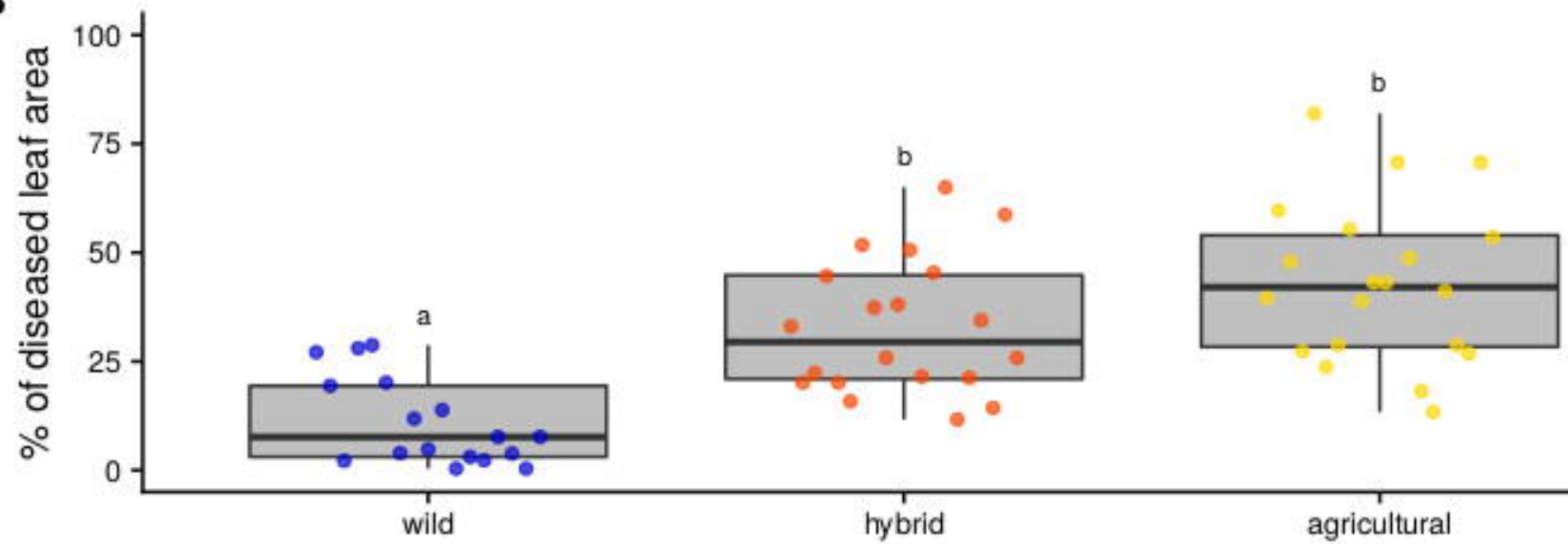

hybrid

agricultural 


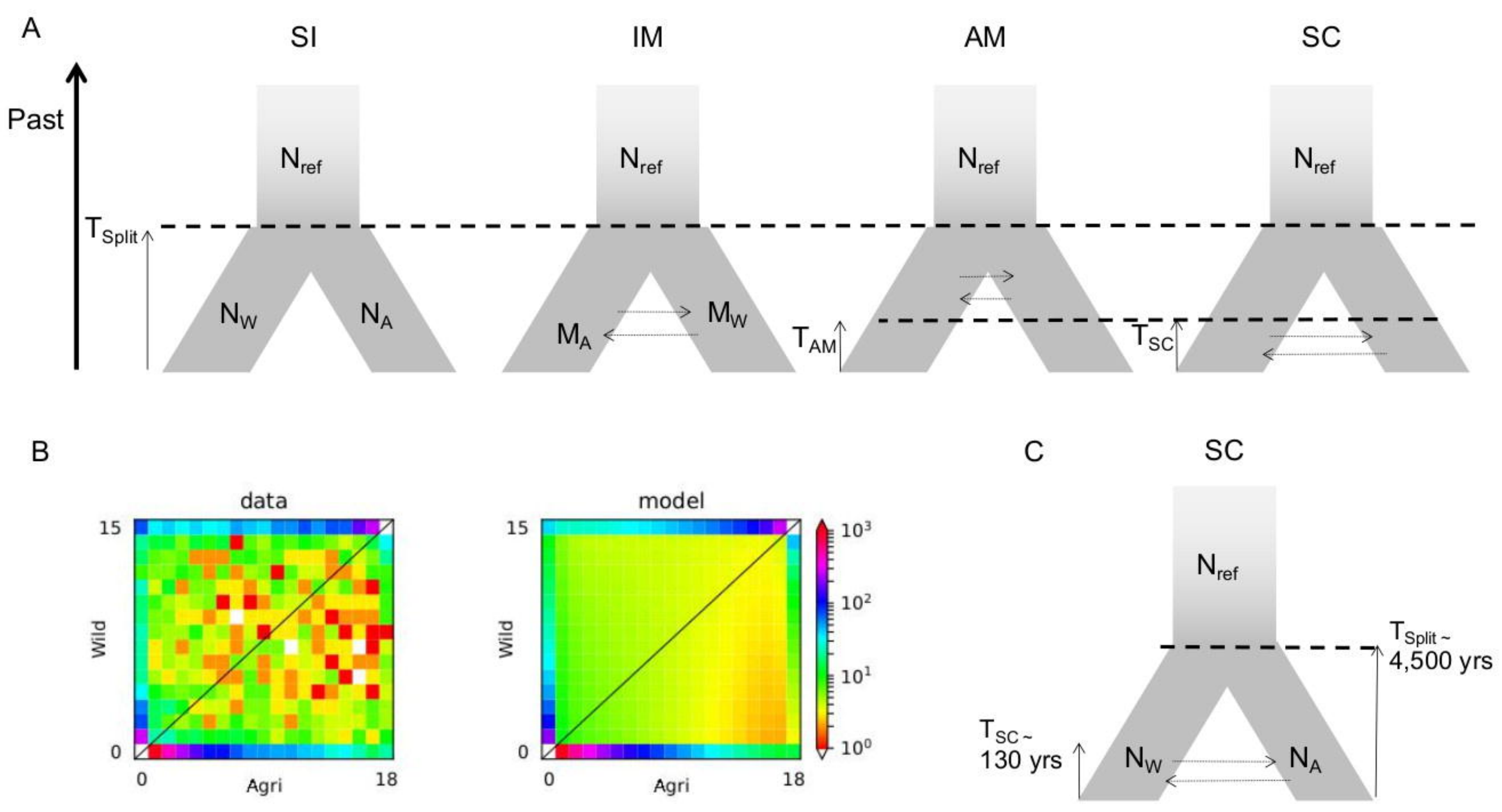

